\title{
Well-Balanced Finite Volume schemes for 2D non-homogeneous hyperbolic systems. Application to the Dam-break of Aznalcóllar.
}

\author{
M. J. Castro Díaz † T. Chacón Rebollo

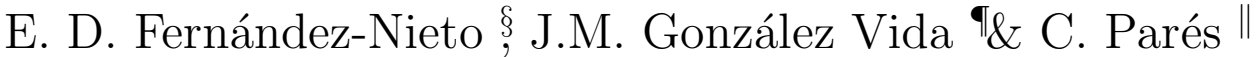

\begin{abstract}
In this paper we introduce a class of well-balanced Finite Volume schemes for 2D non-homogeneous hyperbolic systems. We extend the derivation of standard Finite Volume solvers for homogeneous systems to non-homogeneous ones using the method of lines. We study conservation and some well-balanced properties of the numerical scheme. We apply our solvers to Shallow Water Equations: We prove that these exactly compute the water at rest solutions. We also perform some numerical tests, by comparing with 1D solutions, simulating the formation of a hydraulic drop and a hydraulic jump, and studying a real dam break: Aznalcóllar, an ecological disaster happened in the province of Seville, Spain in 1998.
\end{abstract}

Short title : 2D Asymptotically balanced schemes.

Keywords : Finite Volume Method, well-balanced, upwinding, shallow water, source terms.

Subject Classifications : AMS (MOS) : 65N06, 76B15, 76M20, 76N99.

\footnotetext{
*This research was partially supported by Spanish Government Research projects .....

†Dpto. Análisis Matemático, U. Málaga, Campus Teatinos s/n, Málaga, Spain (castro@anamat.cie.uma.es)

${ }^{\ddagger}$ Dpto. Ecuaciones Diferenciales y Análisis Numérico, U. Sevilla. C/ Tarfia, s/n. 41080 Sevilla, Spain (chacon@us.es).

$\S$ Dpto. Matemática Aplicada I, E.T.S. Arquitectura, U. de Sevilla, Avda. Reina Mercedes 2, 41012 Sevilla, Spain (edofer@us.es).

IDpto. Matemática Aplicada, U. Málaga, Spain (vida@anamat.cie.uma.es)

"Dpto. Análisis Matemático, U. Málaga, Campus Teatinos s/n, Málaga, Spain (pares@anamat.cie.uma.es)
} 


\section{Introduction}

In this paper we deal with the derivation of well-balanced solvers for non-homogeneous 2D hyperbolic systems of conservation laws. Our work is motivated by the need of obtaining efficient solvers of 2D Shallow Water Equations (SWE in what follows) with variable bottom topography, but most of our conclusions also apply to general 2D hyperbolic systems with source terms.

The accurate solution of hyperbolic systems with source terms requires numerical solvers with specific properties. Indeed, an upwind discretization of the source term, compatible with that of the flow term, must be performed. Otherwise, a first error order in space, stemming from the numerical diffusion terms, takes place. This error, after time iteration, may yield large errors in wave amplitude and speed. Roe in [27] studies the relation between the choice of quadrature formulae to approximate the average of the source term and the property of preserving the stationary solutions.

Bermúdez and Vázquez-Céndón introduce in [5] some numerical solvers that upwind the source term for 1D SWE, by preserving water at rest. This work originated the so-called "well-balanced" solvers, in the sense that the discrete source terms balance the discrete flux terms when computed on some (or all) of the steady solutions of the continuous systems. Several sequels of this work for 1D SWE followed. See for example: Castro et al. [13], Greenberg-Leroux [18], Kurganov-Levy [21], Le Veque [22], Perthame-Simeoni [24], Zhou et al. [32].

A different way of studying the numerical treatment of source terms is to rewrite the system as a non-conservative hyperbolic system, by adding up a new equation. For example, for SWE with topography, by introducing that the derivative in time of the bottom function is zero. A numerical scheme for non-conservative hyperbolic system is then applied and finally it is rewritten in terms of the original variables. The main difficulty of non-conservative systems both from the mathematical and the numerical points of view comes from the presence of non-conservative products, which makes difficult even the definition of weak solutions. In [15] authors introduce the definition of non-conservative products as Borel measures given by Dal Maso, LeFloch and Murat. This definition, which depends on the choice of a family of paths in the phase space, allows one to give a rigorous definition of weak solutions for non-conservative hyperbolic systems.

Parés and Castro present in [23] a study of well-balanced properties of Roe method for non-conservative hyperbolic systems. They show that the scheme proposed by Bermudez-Vázquez [5] corresponds to the choice of a family of segments in the definition of Roe linearization.

The paper of Bermúdez and Vázquez-Céndón [4] reported a first extension of Finite Volume schemes to 2D SWE with source terms stemming from variable bottom topography. This extension followed the general idea of upwinding the source terms, once the equation is discretized by means of local balances of fluxes between neighboring cells. An extension of this method to the case of 2D hyperbolic systems with non-conservative products is presented by Castro et al. in [9]. This paper in- 
cludes an application to the bilayer 2D SWE, to simulate the steady exchange flow through the Strait of Gibraltar.

Several authors follow different approaches to discretize the shallow water equations by well-balanced solvers. In particular, in [11] Brufau and García-Navarro derive a multidimensional upwind Finite Volume solver. This distributive scheme is applied to the component of the source term stemming from the bottom gradient. In this way, the water at rest is exactly preserved.

Hervouet uses in [19] Finite Element solvers combined with time discretization by the Method of Characteristics, and an operator splitting technique. The description of the evolution in time of the increment of the column of water in terms of the gradient of the free surface allows to preserve the water at rest solution.

Audusse et al. use in [3] kinetic schemes to approach 2D SWE. With a kinetic formulation, the system becomes a linear equation, where the unknown is the "microscopic density of particles". To deduce the numerical scheme the original unknowns are updated by an averaging process. The collision term in the kinetic equation is neglected, what can be interpreted as performing all collisions at once on each control volume. To achieve the well-balance property for still water flow, new interface topography and water depth values are defined. This scheme has the specific advantage of preserving the water depth positiveness under a rather restrictive CFL condition.

A different approach is followed by Amara, Capatina and Trujillo in [2]. Here, an alternative form of 2D SWE is derived from a variational formulation of 3D NavierStokes equation with free surface. The solution of this alternative form, together with a post-processed vertical velocity, is a particular solution of the 3D NavierStokes equations. Error estimates in terms of the thickness of the water layer are deduced.

Aizinger and Dawson present in [1] a local discontinuous Galerkin method for 3D SWE. The system includes the momentum equation for the horizontal components of the velocity using the hydrostatic pressure, a continuity equation involving the vertical velocity and an equation describing the motion of the free surface. Moreover [1] studies the stability properties, proving the $\mathrm{L}^{2}$ stability for the Local Discontinous Galerkin method for this 3D SWE non-linear model.

In [25] Quecedo et al. present a comparison of Navier-Stokes equations with SWE for solving the dam break problem. The Navier-Stokes equations are discretizated using a Finite Element Method with a Level-Set algorithm and the SWE equations are discretized by using a Taylor-Galerkin scheme. One of the conclusions of the paper of Quecedo et al. is that the SWE approach is appropriate for large computational domains, due to a smaller computational cost. Moreover, the results obtained with SWE and Navier-Stokes equations for the evolution of the water depth far from the dam are close.

The derivation of systematic techniques to build well-balanced numerical schemes is also a relevant issue, as this is far from being a straightforward property to obtain. In [14] a systematic derivation of well-balanced solvers for 1D systems for hyperbolic 
conservation laws is introduced. The main idea is to discretize an equivalent equation with an additional source term. This term is specifically designed to balance the first order term stemming from the numerical diffusion due to the actual numerical scheme for the homogeneous equation.

We focus in this paper on the systematic derivation of well-balanced solvers for $2 \mathrm{D}$ non-homogeneous hyperbolic systems of conservation laws by finite volume methods. To do this we proceed similarly to the derivation of standard finite volume solvers using the line method: we project the system (including the source terms) along the normal to the boundary segment between two neighboring cells, assuming that the source terms only vary along the normal direction. Then, a $1 \mathrm{D}$ well-balanced approximated Riemann solver is applied to solve the resulting problems.

By this procedure we obtain a large family of conservative solvers that we prove to be asymptotically well-balanced for essentially 1D solutions. In addition, we give general conditions that we prove to be sufficient for a given scheme to exactly solve an actual steady solution at grid nodes. These conditions are independent of the numerical diffusion matrix of the scheme, thus largely simplifying the calculations.

We apply this general theory to 2D SWE, for which we introduce a systematic discretization of source terms, in particular those coming from variable bottom topography. We also introduce a large family of schemes that we prove to be asymptotically well-balanced for essentially 1D solutions, and to exactly solve water at rest. We apply one of the solvers introduced in this paper to solve the SWE, for simulating the real break of the dam of Aznalcóllar.

Although at present we are not able to give a proof of stability of our methods or error estimates, in practice these yield quite good accuracy and stability properties in tests involving strong shocks. This is confirmed by the hard test of the real dam break of Aznalcollar.

This paper is organized as follows: In section 2, we summarize the basic technique to discretize 1D hyperbolic systems with source terms. In Section 3, we present a systematic derivation of well-balanced solvers for 2D Finite Volume schemes using the line method. In Section 4, we study the conservation and balance properties of the 2D proposed schemes. In Section 5 we present the application of the proposed solvers to SWE. We also prove that the schemes exactly balance the stationary solution of water at rest. In Section 6 we present three numerical tests. The first one is a $1 \mathrm{D}$ double rarefaction wave with vacuum. The second test is the simulation of the formation of an hydraulic jump and an hydraulic drop due to a supercritical flow facing a descending ramp. The third test is an application to the study of the flood produced by the dam break of the mining ponds of Aznalcóllar, a real ecological disaster happened in Spain in 1998. Finally, in Section 7 we present the conclusions of the paper. 


\section{Balanced schemes for 1D non-homogeneous sys- tems}

In this section we study the discretization of $1 \mathrm{D}$ non-homogeneous hyperbolic systems of conservation laws. We shall summarize the derivation of a well-balanced family of schemes for these systems introduced in [14]. We shall use this derivation to construct well-balanced Finite Volume schemes for 2D non-homogeneous hyperbolic systems in the next section.

We consider systems with the structure

$$
\begin{cases}\frac{\partial W(x, t)}{\partial t}+\frac{\partial}{\partial x} F(W(x, t))=G(x, W(x, t)) & x \in] 0, L[, \quad t \in] 0, T[, \\ W(x, 0)=W_{0}(x) & x \in[0, L],\end{cases}
$$

where $W:[0, L] \rightarrow \mathbf{R}^{N}$ is the unknown function, $F: \mathbf{R}^{N} \rightarrow \mathbf{R}^{N}$ is the physical flux function and $G:[0, L] \times \mathbf{R}^{N} \rightarrow \mathbf{R}^{N}$ is the source term. We will suppose that $F$ and $G$ are known piecewise $C^{2}$ functions.

We consider a partition $\left\{x_{i}\right\}_{i=0}^{M+1}$ of the interval $[0, L]$, with step $\Delta x$, and a partition $\left\{t^{n}\right\}_{n=0}^{M+1}$ of $[0, T]$. We also denote $x_{i+1 / 2}=\left(x_{i}+x_{i+1}\right) / 2$ for $i=0, \ldots, M$.

We consider finite volume schemes that can be written in conservative form, for the space discretization. To discretize in time, we use the explicit Euler scheme.

In the homogeneous case, $G=0$, we consider conservative schemes with the structure

$$
W_{i}^{n+1}=W_{i}^{n}-\frac{\Delta t}{\Delta x}\left(\phi\left(W_{i}^{n}, W_{i+1}^{n+1}\right)-\phi\left(W_{i-1}^{n}, W_{i}^{n}\right)\right),
$$

where $\phi\left(W_{i}, W_{i+1}\right)$ is the numerical flux function. More specifically, we are interested in schemes that can be written in "viscous form", for which the numerical flux $\phi$ has the structure

$$
\phi\left(W_{i}, W_{i+1}\right)=F_{C}\left(W_{i}, W_{i+1}\right)-\frac{1}{2} D\left(W_{i}, W_{i+1}\right)\left(W_{i+1}-W_{i}\right),
$$

where by $F_{C}\left(W_{i}, W_{i+1}\right)$ we denote a centered approximation of $F$ at $x_{i+1 / 2}$, for instance

$$
F_{C}\left(W_{i}, W_{i+1}\right)=\frac{F\left(W_{i}\right)+F\left(W_{i+1}\right)}{2} \quad \text { or } \quad F_{C}\left(W_{i}, W_{i+1}\right)=F\left(\frac{W_{i}+W_{i+1}}{2}\right) .
$$

Also by $D\left(W_{i}, W_{i+1}\right)$ we denote the upwinding matrix of the scheme. This matrix is an approximation of a numerical diffusion matrix $\mathcal{D}\left(\widetilde{W}_{i+1 / 2}\right)$, where $\widetilde{W}_{i+1 / 2}$ is an intermediate state for $W_{i}$ and $W_{i+1}$, for instance its half value or the intermediate state of Roe (Cf. Roe [28]). Matrix $\mathcal{D}$ must be defined (or, at least, semi-defined) positive. It is related to the upwinding matrix by $D(W, W)=\mathcal{D}(W)$.

We suppose that $\mathcal{D}(W)$ is a Lipschitz function of $W$. Its derivatives can have some discontinuities at points where some of its eigenvalues vanish. Some examples 
of matrices $D$ are the absolute value $|A|$ (Roe scheme), $\lambda A^{2}$ (Lax-Wendroff scheme), a combination of Roe and Lax-Wendroff methods with flux limiters, or a diagonal matrix (Lax-Friedrichs scheme).

The derivation of the well-balanced family of schemes introduced in [14] starts from the observation that numerical schemes written in viscous form and defined by (2), (3) can be interpreted as centered discretization of the parabolic equivalent system

$$
\frac{\partial W}{\partial t}+\frac{\partial}{\partial x} F(W)-\nu \frac{\partial}{\partial x}\left(\mathcal{D}(W) \frac{\partial}{\partial x} W\right)=0
$$

where $\nu$ is equal to half of the space step $(\nu=\Delta x / 2)$ and $\mathcal{D}(W)$ is the numerical diffusion matrix. All stationary solutions of the hyperbolic homogeneous system, when $A(W)$ is inversible, are constant and, then, also are stationary solutions of the equivalent system (5).

In the case of the hyperbolic system with source term,

$$
\frac{\partial W}{\partial t}+\frac{\partial}{\partial x} F(W)=G(x, W)
$$

a "viscous correction" of the source term,

$$
\frac{\partial W}{\partial t}+\frac{\partial}{\partial x} F(W)-\nu \frac{\partial}{\partial x}\left(\mathcal{D}(W) \frac{\partial}{\partial x} W\right)=G(x, W)+C(W) .
$$

is proposed to compensate for the numerical diffusion introduced by the discretization of the physical flux on steady solutions.

If the Jacobian matrix $A$ of the flux $F$ is non singular at $W$, the correcting term is defined as

$$
C(W)=-\nu \frac{\partial}{\partial x}\left(\mathcal{D}(W) A^{-1}(W) G(x, W)\right) .
$$

In this way, (7) is rewritten as

$$
\frac{\partial W}{\partial t}+\frac{\partial}{\partial x}\left[F(W)-\nu \mathcal{D}(W) \frac{\partial}{\partial x} W\right]=G(x, W)-\nu \frac{\partial}{\partial x}\left[\mathcal{D}(W) A^{-1}(W) G(x, W)\right]
$$

It is concluded that to build balanced numerical schemes for the non-homogeneous hyperbolic system (6) it is enough to use second order approximations in space of (9). Indeed, a stationary solution $\bar{W}$ of (6) verifies

$$
A(\bar{W}) \frac{\partial \bar{W}}{\partial x}=\frac{\partial}{\partial x} F(\bar{W})=G(x, \bar{W}) .
$$

Therefore, if $A(\bar{W})$ is non-singular,

$$
\frac{\partial \bar{W}}{\partial x}=A^{-1}(\bar{W}) G(x, \bar{W})
$$


and the diffusion flow and source terms in (9) balance each other.

Moreover, these schemes are built with conservative structure. Indeed, (9) can be re-written as

$$
\frac{\partial W}{\partial t}+\frac{\partial}{\partial x}\left[F(W)-\nu \mathcal{D}(W)\left(\frac{\partial W}{\partial x}-A^{-1}(W) G(x, W)\right)\right]=G(x, W) .
$$

This equation is discretized with three-points schemes with the structure

$$
\begin{gathered}
\frac{W_{i}^{n+1}-W_{i}^{n}}{\Delta t}+\frac{\phi_{G}\left(W_{i}^{n}, W_{i+1}^{n}\right)-\phi_{G}\left(W_{i-1}^{n}, W_{i}^{n}\right)}{\Delta x}= \\
=G_{C}\left(x_{i-1}, x_{i}, x_{i+1}, W_{i-1}^{n}, W_{i}^{n}, W_{i+1}^{n}\right)
\end{gathered}
$$

where by $\phi_{G}$ we denote the numerical flux function modified by the presence of the source term. By $G_{C}$ we denote a centered approximation of $G$ at $x=x_{i}$.

The modified flux $\phi_{G}$ is defined as

$$
\begin{gathered}
\phi_{G}\left(W_{i}, W_{i+1}\right)=F_{C}\left(W_{i}, W_{i+1}\right)- \\
-\nu D\left(W_{i}, W_{i+1}\right)\left(\frac{W_{i+1}-W_{i}}{\Delta x}-\widetilde{A^{-1}}\left(W_{i}, W_{i+1}\right) G_{D}\left(x_{i}, x_{i+1}, W_{i}, W_{i+1}\right)\right) .
\end{gathered}
$$

Here, $G_{D}$ is an approximation of $G$ at $x=x_{i+1 / 2}$ and $\widetilde{A^{-1}}\left(W_{i}, W_{i+1}\right)$ is an approximation of $A^{-1}\left(\widetilde{W}_{i+1 / 2}\right)$. We also remember that $D\left(W_{i}, W_{i+1}\right)$ is an approximation of $\mathcal{D}\left(\widetilde{W}_{i+1 / 2}\right)$ and $\widetilde{W}_{i+1 / 2}$ is an intermediate state for the values $W_{i}$ and $W_{i+1}$.

To approximate $A^{-1}(W)$ when $A(W)$ is singular, this matrix is replaced by the matrix $\widetilde{A^{-1}}(W)$ defined as:

If $A=X \Lambda X^{-1}$, with $\Lambda=\operatorname{Diag}\left(\lambda_{j}, j=1, \ldots, N\right)$, then $\widetilde{A^{-1}}=X \widetilde{\Lambda^{-1}} X^{-1}$, where $\widetilde{\Lambda^{-1}}=\operatorname{Diag}\left(\widetilde{\lambda_{j}^{-1}}, j=1, \ldots, N\right) \quad$ with $\quad \widetilde{\lambda_{j}^{-1}}=\left\{\begin{array}{ccc}1 / \lambda_{j} & \text { if } & \lambda_{j} \neq 0 \\ 0 & \text { if } & \lambda_{j}=0\end{array} \quad i=1, \ldots, N\right.$

The idea underlying this definition is that when some eigenvalue of $A$ vanishes, then no upwinding of the corresponding characteristic field is needed. Then, no upwinding of the corresponding component of the source term is neither necessary. This definition of $\widetilde{A^{-1}}$ also allows to balance the scheme in some situations where $A(W)$ is singular in sets with nonzero measure.

Remark 1 Observe that even centered flux and source terms discretizations should be compatible.

Remark 2 Under this point of view, the generalization of the proposed $1 D$ numerical schemes to $2 D$ systems is very simple and natural, by using analogous techniques as for homogeneous systems. It is important to start from 1D numerical schemes written under the conservative structure (11), this allows to introduce a systematic technique to generalize $1 D$ solvers for non-homogeneous hyperbolic systems to $2 D$. 
Another form to study the discretization of $1 D$ systems can be seen for example in [5], in this paper the authors propose to preserve the structure of the numerical flux function of the homogeneous problem and to introduce an upwind discretization of the source term. In fact, the schemes presented in [5] can be easily rewritten under the structure (11).

These schemes balance a large class of stationary solutions in all $[0, L]$ but on a set whose measure tends to zero as $\Delta x \rightarrow 0$. These schemes are called "asymptotically balanced" (Cf. [14]).

DEFINITION 1 (Asymptotically balanced schemes)

We say that scheme (11)-(12) is asymptotically balanced for a stationary solution $\bar{W}(x)$ of the hyperbolic system (1) if there is an increasing sequence of compact sets $\left\{K_{n}\right\}_{n} \subset[0, L]$ such that

1) $\mu\left([0, L] \backslash \cup_{n} K_{n}\right)=0$, where $\mu$ denotes the Lebesgue measure in $\mathbf{R}$.

2) For all $n$ there exists a value $\delta_{n}>0$ such that if $0<\Delta x<\delta_{n}$, the scheme balances the system in $K_{n}$ up to second order.

It seems hopeless to expect that these schemes balance all stationary solutions in all the domain $] 0, L[$ up to second order of accuracy, as the diffusion matrix $\mathcal{D}(W)$ is only Lipschitz-continuous and the additional term $C(W)$ presents singularities when some eigenvalues of $A$ change sign.

However, in the case of SWE, many of these schemes exactly balance some stationary solutions at the nodes of the mesh; in particular water at rest.

We shall precise these statements in Section 5, where we analyze the wellbalanced properties of these schemes for 2D Finite Volume methods that we introduce in the next section.

\section{Finite Volume schemes for 2D systems.}

Let us now consider a general hyperbolic system of conservation laws with source terms in two space dimensions on a bounded domain $\Omega \subset \mathbf{R}^{2}$,

$$
\begin{cases}\frac{\partial W}{\partial t}+\frac{\partial}{\partial x} F_{1}(W)+\frac{\partial}{\partial y} F_{2}(W)=G(x, W) & \text { in } \Omega \times] 0, T[ \\ W(x, 0)=W_{0}(x) & \text { in } \Omega\end{cases}
$$

completed with suitable boundary conditions on $\partial \Omega \times] 0, T[$.

Here, $W: \Omega \times\left[0,+\infty\left[\rightarrow \mathbf{R}^{d}\right.\right.$ is the unknown function; $F_{1}, F_{2}: \mathbf{R}^{d} \rightarrow \mathbf{R}^{d}$ are the flux functions, and $G: \Omega \times \mathbf{R}^{d} \rightarrow \mathbf{R}^{d}$ is the source term. We assume that $F_{1}$, $F_{2}$ and $G$ are known functions with piecewise $C^{2}$ regularity.

Let us also consider a partition of $\Omega$ (assumed to be polygonal) $\mathcal{V}_{h}=\left\{V_{i}\right\}_{i=1}^{n}$ into control volumes. We shall denote by $\Gamma_{i j}$ the interface between two adjacent control volumes $V_{i}$ and $V_{j}$, and by $\eta_{i j}$ its normal pointing from $V_{i}$ to $V_{j}$. For each control 
volume $V_{i}$ we denote by $K_{i}$ the set of indices of the neighboring control volumes. If $\Gamma$ is a straight segment of $\partial V_{i}$ located on $\partial \Omega$, we add to $\mathcal{V}_{h}$ a virtual control volume $V_{j}$, adjacent to $\Gamma$ and exterior to $\Omega$. We still denote $\Gamma$ by $\Gamma_{i j}$. This is a useful formalism to impose the boundary conditions.

Integrating (14) on $V_{i}$,

$$
\int_{V_{i}} \frac{\partial W}{\partial t}+\sum_{j \in K_{i}} \int_{\Gamma_{i j}} \mathbb{F}(W) \cdot \eta_{i j}=\int_{V_{i}} G(x, W) \mathrm{d} x,
$$

where we denote $\mathbb{F}=\left(F_{1}, F_{2}\right)$. Following the basic idea of Finite Volume schemes for the homogeneous case, we approximate this identity by

$$
\left|V_{i}\right| \frac{W_{i}^{n+1}-W_{i}^{n}}{\Delta t}+\sum_{j \in K_{i}}\left\|\Gamma_{i j}\right\| \phi_{G}\left(W_{i}^{n}, W_{j}^{n}, \eta_{i j}\right)=\left|V_{i}\right| G_{i}^{n} .
$$

Here, $W_{i}^{n}$ represents an approximation to the average $\frac{1}{\left|V_{i}\right|} \int_{V_{i}} W\left(x, t_{n}\right) \mathrm{d} x, G_{i}^{n}$ is an approximation of $\frac{1}{\left|V_{i}\right|} \int_{V_{i}} G\left(x, W\left(x, t_{n}\right)\right) \mathrm{d} x$, and $\phi_{G}$ is some numerical flux function associated to the projection of system (14) on the direction $\eta_{i j}$. To construct this projection, let us consider a vector $\eta \in \mathbf{R}^{2}$.

Observe that

$$
\begin{aligned}
\frac{\partial}{\partial x} F_{1}(W) & +\frac{\partial}{\partial y} F_{2}(W)=\sum_{i=1}^{2}\left\{\partial_{i} F_{1}(W) \eta_{1}+\partial_{i} F_{2}(W) \eta_{2}\right\}[\nabla W]_{i} \cdot \eta+ \\
& +\sum_{i=1}^{2}\left\{-\partial_{i} F_{1}(W) \eta_{2}+\partial_{i} F_{2}(W) \eta_{1}\right\}[\nabla W]_{i} \cdot \eta^{\perp}
\end{aligned}
$$

where we denote $[\nabla W]_{i}=\left(\partial_{1} W_{i}, \partial_{2} W_{i}\right)$ and $\eta^{\perp}=\left(-\eta_{2}, \eta_{1}\right)$. Indeed,

$$
\begin{array}{r}
\sum_{i=1}^{2}\left\{\partial_{i} F_{1}(W) \eta_{1}\right\}[\nabla W]_{i} \cdot \eta+\sum_{i=1}^{2}\left\{-\partial_{i} F_{1}(W) \eta_{2}\right\}[\nabla W]_{i} \cdot \eta^{\perp}= \\
\sum_{i=1}^{2} \partial_{i} F_{1}(W)\left[\eta_{1}^{2} \partial_{1} W_{i}+\eta_{1} \eta_{2} \partial_{2} W_{i}+\eta_{2}^{2} \partial_{1} W_{i}-\eta_{1} \eta_{2} \partial_{2} W_{i}\right]= \\
\sum_{i=1}^{2} \partial_{i} F_{1}(W)\left(\eta_{1}^{2}+\eta_{2}^{2}\right) \partial_{1} W_{i} .
\end{array}
$$

Similarly,

$$
\begin{array}{r}
\sum_{i=1}^{2}\left\{\partial_{i} F_{2}(W) \eta_{2}\right\}[\nabla W]_{i} \cdot \eta+\sum_{i=1}^{2}\left\{\partial_{i} F_{2}(W) \eta_{1}\right\}[\nabla W]_{i} \cdot \eta^{\perp}= \\
\sum_{i=1}^{2} \partial_{i} F_{2}(W)\left(\eta_{1}^{2}+\eta_{2}^{2}\right) \partial_{1} W_{i}
\end{array}
$$




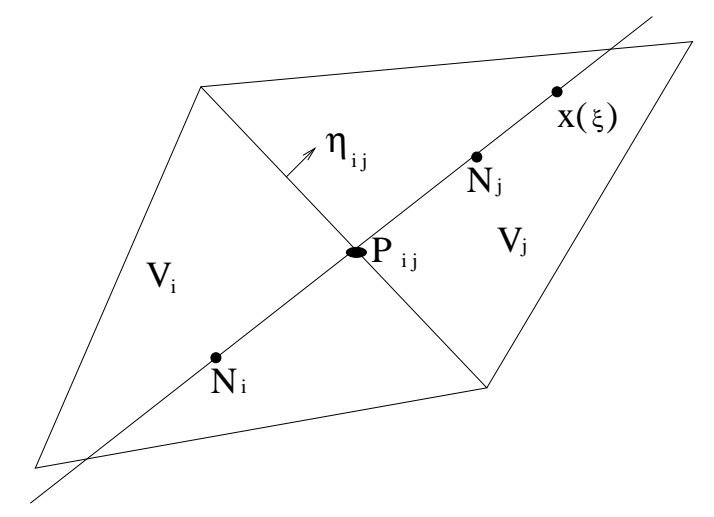

Figure 1: Definition of $N_{i}, P_{i j}$ and the line $x(\xi)$

Assume that a solution $W(x, t)$ of (14) does not vary in the direction $\eta^{\perp}=$ $\left(-\eta_{2}, \eta_{1}\right)$, that is

$$
\nabla W \cdot \eta^{\perp}=0 .
$$

If we define the $1 \mathrm{D}$ vector function $U(\xi, t)=W(x(\xi), t)$ with $x(\xi)=\xi \eta+x_{0}$ (for some $\left.x_{0} \in \mathbf{R}^{2}\right)$, then $\frac{\partial}{\partial \xi} U(\xi, t)=\nabla W(x(\xi), t) \cdot \eta$ and

$$
\begin{gathered}
\frac{\partial}{\partial \xi}\left[F_{1}(U) \eta_{1}+F_{2}(U) \eta_{2}\right](\xi, t)=\sum_{i=1}^{d}\left\{\partial_{i} F_{1}(U) \eta_{1}+\partial_{i} F_{2}(U) \eta_{2}\right\}[\nabla W]_{i}(x(\xi), t) \cdot \eta= \\
=\left[\frac{\partial}{\partial x} F_{1}(W)+\frac{\partial}{\partial y} F_{2}(W)\right](x(\xi), t)
\end{gathered}
$$

Consequently, $U(\xi, t)$ is a solution of the $1 \mathrm{D}$ hyperbolic system with source term

$$
\frac{\partial U}{\partial t}+\frac{\partial}{\partial \xi}[(\mathbb{F} \cdot \eta)(U)]=\widetilde{G}(\xi, U)
$$

where $\mathbb{F}=\left(F_{1}, F_{2}\right)$ and $\widetilde{G}(\xi, U)=G(x(\xi), U)$.

This suggests to define $\phi_{G}\left(W_{i}, W_{j}, \eta_{i j}\right)$ as some approximation of the flux at $\xi=0$ of the solution for the 1D non-homogeneous Riemann problem

$$
\left\{\begin{array}{l}
\left.\frac{\partial U}{\partial t}+\frac{\partial}{\partial \xi}[(\mathbb{F} \cdot \eta)(U)]=\widetilde{G}_{i j}(\xi, U) \text { in } \mathbf{R} \times\right] 0,+\infty[, \\
U(\xi, 0)= \begin{cases}W_{i} & \text { if } \xi<0, \\
W_{j} & \text { if } \xi>0 ;\end{cases}
\end{array}\right.
$$

where $\widetilde{G}_{i j}(\xi, U)=G\left(P_{i j}+\xi \eta_{i j}, U\right)$, for some point $P_{i j} \in \Gamma_{i j}$ (see Figure 1). To obtain balanced $2 \mathrm{D}$ schemes, we use the fluxes for balanced 1D schemes defined in the previous section. 
Let us define $\phi_{G}$. We first observe that using (20), the Jacobian matrix of the $1 \mathrm{D}$ flux $\mathbb{F} \cdot \eta$ is

$$
\mathcal{A}(U, \eta)=F_{1}^{\prime}(U) \eta_{1}+F_{2}^{\prime}(U) \eta_{2}
$$

Next, we identify the cell values $W_{i}$ and $W_{j}$ with nodal values at some points $N_{i} \in V_{i}$ and $N_{j} \in V_{j}$ located along the straight $x(\xi)=P_{i j}+\xi \eta_{i j}$.

Then, we set

$$
\begin{gathered}
\phi_{G}\left(W_{i}, W_{j}, \eta_{i j}\right)=\mathbb{F}_{C}\left(W_{i}, W_{j}, \eta_{i j}\right)- \\
-\nu_{i j} D\left(W_{i}, W_{j}, \eta_{i j}\right)\left(\frac{W_{j}-W_{i}}{d_{i j}}-\widetilde{A^{-1}}\left(W_{i}, W_{j}, \eta_{i j}\right) \widehat{G}_{i j}\left(W_{i}, W_{j}, \eta_{i j}\right)\right)
\end{gathered}
$$

where $\mathbb{F}_{C}$ is a second order approximation of $\mathbb{F}\left(\frac{W_{i}+W_{j}}{2}\right) \cdot \eta ; d_{i j}$ is the distance between $N_{i}$ and $N_{j} ; \nu_{i j}=d_{i j} / 2 ; \widetilde{A^{-1}}\left(W_{i}, W_{j}, \eta_{i j}\right)$ is some first order approximation of $\widetilde{A^{-1}}\left(\frac{W_{i}+W_{j}}{2}, \eta_{i j}\right)$, and $\widehat{G}_{i j}\left(W_{i}, W_{j}, \eta_{i j}\right)$ is some first order approximation of $G\left(P_{i j}, \frac{W_{i}+W_{j}}{2}\right)$.

Then, by using the numerical flux modified by the source term we can easily apply the same type of extension as in the case of homogeneous system. We obtain the scheme defined by (15)-(22).

\section{Conservation and well-balanced properties}

In this section we prove the conservation and the balance properties of the proposed scheme.

\subsection{Conservation}

We start by analyzing the conservation properties by a rather general result.

THEOREM 1 Let us denote by $W_{h}^{n}: \bar{\Omega} \rightarrow \mathbf{R}^{d}$ an approximation of $W\left(x, t_{n}\right)$ built by means of the discrete values $\left\{W_{i}^{n}\right\}$. We suppose that

a) $W_{h}^{n}$ verifies $W_{i}^{n}=\frac{1}{\left|V_{i}\right|} \int_{V_{i}} W_{h}^{n}(\mathbf{x}) d \mathbf{x}, G_{i}^{n}=\frac{1}{\left|V_{i}\right|} \int_{V_{i}} G\left(\mathbf{x}, W_{h}^{n}(\mathbf{x})\right) d \mathbf{x}$.

b) The diffusive flux vanishes on the boundary of $\Omega(D=0$ on $\partial \Omega)$.

c) The following symmetries hold,
c1) $\mathbb{F}_{C}(U, V, \eta)=-\mathbb{F}_{C}(V, U,-\eta)$
c2) $D(U, V, \eta)=D(V, U,-\eta)$
c3) $\widetilde{A^{-1}}(U, V, \eta)=-\widetilde{A^{-1}}(V, U,-\eta)$
c4) $\widehat{G}(U, V, \eta)=\widehat{G}(V, U,-\eta)$ 
Then, the scheme defined by (15)-(22) is globally conservative, in the sense that

$$
\begin{aligned}
\frac{\int_{\Omega} W_{h}^{n+1}(\mathbf{x}) d \mathbf{x}-\int_{\Omega} W_{h}^{n}(\mathbf{x}) d \mathbf{x}}{\Delta t} & +\sum_{\Gamma_{i j} \subset \partial \Omega}\left\|\Gamma_{i j}\right\| \mathbb{F}_{C}\left(W_{i}^{n}, W_{j}^{n}, \eta_{i j}\right) \\
& =\int_{\Omega} G\left(\mathbf{x}, W_{h}^{n}(\mathbf{x})\right) d \mathbf{x} .
\end{aligned}
$$

Proof: Due to $c 1)-c 4$ ), the function $\phi_{G}$ defined by (22) verifies

$$
\phi_{G}(U, V, \eta)=-\phi_{G}(V, U,-\eta)
$$

Now, summing in equation (15), all contributions from $\phi_{G}$ due to internal faces $\Gamma_{i j}$ vanish. So we recover

$$
\sum_{i=1}^{n}\left|V_{i}\right| \frac{W_{i}^{n+1}-W_{i}^{n}}{\Delta t}+\sum_{\Gamma_{i j} \subset \partial \Omega}\left\|\Gamma_{i j}\right\| \phi_{G}\left(W_{i}^{n}, W_{j}^{n}, \eta_{i j}\right)=\sum_{i=1}^{n}\left|V_{i}\right| G_{i}^{n} .
$$

Using now hypothesis $a$ ) and $b$ ) we conclude (23).

We observe that if $G_{i}$ is an approximation of order $\mathcal{O}\left(h^{k}\right)$ (where $h$ is the diameter of the partition) of $\frac{1}{\left|V_{i}\right|} \int_{V_{i}} G(x, W(x)) \mathrm{d} x$, the method verifies (23) up to order $h^{k}$.

Conditions $c 1$ ) to $c 4$ ) are all natural, linked to the symmetries of problem (21) with respect to exchanging $W_{i}$ and $W_{j}$.

We observe that Hypotheses $c 1$ ) and $c 2$ ) ensure that the numerical flux function of the homogeneous hyperbolic system is conservative, that is, $\phi(U, V, \eta)=$ $-\phi(V, U,-\eta)$. Hypothesis $c 3)$ and $c 4)$ are additional sufficient conditions to ensure that

$$
\phi_{G}(U, V, \eta)=-\phi_{G}(V, U,-\eta)
$$

\subsection{Balance properties}

In this subsection we study the balance properties of the proposed method. We study wether it verifies the same properties as a $2 \mathrm{D}$ finite volume method for the homogeneous case: by the form in which it is built, we study 1D solutions discretized on a mesh that verifies hypothesis (19).

We are able to prove an asymptotic balance property for essentially one-dimensional solutions. We also give sufficient conditions for a scheme to exactly balance a given stationary solution at grid nodes. These conditions are simple to verify.

In practice, our scheme solves quite accurately, combined with mesh-refinement techniques, all kind of solutions. We present some numerical evidence of this fact in the numerical tests section.

We start by setting the following definitions: 
DEFINITION 2 We say that a solution $W(\mathbf{x}, t)$ of system (14) is essentially $1 D$ along the direction $\eta \in \mathbf{R}^{2}$ if there exists a $1 D$ function $U:[E, F] \times[0, T] \rightarrow \mathbf{R}$ such that

$$
W(\mathbf{x}, t)=U(\xi(\mathbf{x}), t)
$$

with $\xi(\mathbf{x})=\left(\mathbf{x}-\mathbf{x}_{0}\right) \cdot \eta+\xi_{0}$ for some $\mathbf{x}_{0} \in \mathbf{R}^{2}, \xi_{0} \in \mathbf{R}$, provided

$$
G(\mathbf{x}, W(\mathbf{x}, t))=\widetilde{G}(\xi(\mathbf{x}), U(\xi(\mathbf{x}), t))
$$

for some function $\widetilde{G}: \mathbf{R} \times \mathbf{R}^{N} \rightarrow \mathbf{R}^{N}$.

DeFinition 3 Given a solution $W(\mathbf{x}, t)$ of system (14) essentially $1 D$ along the direction $\eta$, we say that a rectangular grid is compatible with the direction $\eta$ if it can be obtained by a rotation from a cartesian grid in such a way that the positive $x$ axis is mapped into the vector $\eta$.

DEFINITION 4 We shall say that a Finite Volume scheme is directionally asymptotically well-balanced for system (14) if it is asymptotically well-balanced at least with order two for essentially $1 D$ steady solutions, on rectangular grids compatible with the direction of the solution.

With these definitions we may state our main result:

Theorem 2 Assume the hypotheses of Theorem 1 to hold. Assume that the Finite Volume scheme (15)-(22) is built from an asymptotically well-balanced at least with order two $1 D$ scheme. Then, scheme (15)-(22) is directionally asymptotically wellbalanced for system (14).

Proof: Due to property (24) and the essentially 1D nature of $W$, it is straightforward to prove that scheme (15)-(22) reduces to the $1 \mathrm{D}$ scheme (11)-(12), if we identify the initial conditions by $U_{i}^{0}=U\left(\xi_{i}, t^{0}\right)=W_{i}^{0}$.

Now, due to (20) we have

$$
\begin{gathered}
\frac{\partial W}{\partial t}(\mathbf{x}, t)+[\nabla \cdot \mathbb{F}(W)](\mathbf{x}, t)-G(\mathbf{x}, W(\mathbf{x}, t))= \\
=\frac{\partial U}{\partial t}(\xi(\mathbf{x}), t)+\frac{\partial}{\partial \xi}[(\mathbb{F} \cdot \eta)(U)](\xi(\mathbf{x}), t)-\widetilde{G}(\xi(\mathbf{x}), U(\xi(\mathbf{x}), t)) .
\end{gathered}
$$

If we denote

$$
T_{2 D}(W)(\mathbf{x}, t)=\frac{\partial W}{\partial t}(\mathbf{x}, t)+[\nabla \cdot \mathbb{F}(W)](\mathbf{x}, t)-G(\mathbf{x}, W(\mathbf{x}, t))
$$

and

$$
T_{1 D}(U)(\xi, t)=\frac{\partial U}{\partial t}(\xi, t)+\frac{\partial}{\partial \xi}[(\mathbb{F} \cdot \eta)(U)](\xi, t)-\widetilde{G}(\xi, U(\xi, t))
$$


by equality (25) we have that

$$
T_{2 D}(W)(\mathbf{x}, t)=T_{1 D}(U)(\xi(\mathbf{x}), t) .
$$

In what follows we suppose that $W$ is a stationary solution. Moreover, we write as $T_{h, 1 D}\left(U_{h}\right)=0$ and $T_{h, 2 D}\left(W_{h}\right)=0$ the numerical schemes for the one-dimensional system $T_{1 D}(U)=0$ and the two-dimensional system $T_{2 D}(W)=0$, respectively.

We denote by $x_{i j}$ the nodes of the rectangular mesh of $\Omega$, where the index $i$ (for a fixed index level $\mathrm{j}$ ) correspond to nodes along direction $\eta$ and index $j$ (for a fixed index level i) along direction $\eta^{\perp}$. As this mesh is compatible with the direction $\eta$, the values $\xi=x_{i j} \cdot \eta$ are equal for all $j$. We shall suppose that $\Omega$ is the rectangle $\Omega=] A, B[\eta+] C, D\left[\eta^{\perp}\right.$.

Then, if the one dimensional scheme is balanced for $U$ on a compact set $K$, we have

$$
T_{h 1 D}(U)\left(\xi_{i}\right)=T_{1 D}(U)\left(\xi_{i}\right)+\mathcal{O}\left(h^{2}\right),
$$

for all the $1 \mathrm{D}$ nodes, $\xi_{i} \in K$; where $h$ is the space step of the mesh $\left\{\xi_{i}\right\}_{i}$.

Moreover, as the 2D scheme (15)-(22) reduces to the $1 \mathrm{D}$ scheme (11)-(12), we have

$$
T_{h, 2 D}(W)\left(\mathbf{x}_{i}\right)=T_{h, 1 D}(U)\left(\xi_{i}\right), \quad \forall i .
$$

Thus, by (26), (27) and (28) we conclude

$$
\begin{gathered}
T_{h, 2 D}(W)\left(\mathbf{x}_{i}\right)=T_{h, 1 D}(U)\left(\xi_{i}\right)=T_{1 D}(U)\left(\xi_{i}\right)+\mathcal{O}\left(h^{2}\right)=T_{2 D}(W)\left(\mathbf{x}_{i}\right)+\mathcal{O}\left(h^{2}\right), \\
\forall \mathbf{x}_{i} \in \widehat{K}=K \eta+[C, D] \eta^{\perp} .
\end{gathered}
$$

Now, as we suppose that the 1D scheme is asymptotically well-balanced at least with order two, we consider a family of increasing compact sets $\left\{K_{n}\right\} \subset[A, B]$ such that the 1D measure of $[A, B] \backslash \cup_{n} K_{n}$ is zero, and on each compact set $K_{n}$ we have (27) for $0<h<\delta_{n}$.

If we set $\widehat{K_{n}}=K_{n} \eta+[C, D] \eta^{\perp}$, the family $\left\{\widehat{K_{n}}\right\}_{n}$ verifies an analogous property with respect to $\Omega$, and by (29), the system (14) is balanced on each $\widehat{K_{n}}$ if $h<\delta_{n}$.

Moreover, we can give sufficient conditions to exactly balance a given 2D stationary solution at grid nodes. We have the next result:

THEOREM 3 Given a stationary solution $\bar{W}$ of the non-homogeneous hyperbolic system (14), if $\mathbb{F}_{C}\left(\bar{W}_{i}, \bar{W}_{j}, \eta_{i, j}\right), G_{i}$ and $\widehat{G}_{i j}\left(\bar{W}_{i}, \bar{W}_{j}, \eta_{i, j}\right), \forall j \in K_{i}$ verify

$$
\begin{gathered}
\frac{1}{\left|V_{i}\right|} \sum_{j \in K_{i}}\left\|\Gamma_{i j}\right\| \mathbb{F}_{C}\left(\bar{W}_{i}, \bar{W}_{j}, \eta_{i, j}\right)=G_{i}, \\
\frac{\bar{W}_{j}-\bar{W}_{i}}{d_{i j}}=\widetilde{\mathcal{A}^{-1}}\left(\bar{W}_{i}, \bar{W}_{j}, \eta_{i, j}\right) \widehat{G}_{i j}\left(\bar{W}_{i}, \bar{W}_{j}, \eta_{i, j}\right)
\end{gathered}
$$

then the numerical scheme defined by (15)-(22) exactly balances system (14) for the stationary solution. 
Proof: To prove that the scheme exactly balances the stationary solution $\bar{W}$, it is enough to prove

$$
\frac{1}{\left|V_{i}\right|} \sum_{j \in K_{i}}\left\|\Gamma_{i, j}\right\| \phi_{G}\left(\bar{W}_{i}, \bar{W}_{j}, \eta_{i, j}\right)=G_{i} .
$$

But, as $\phi_{G}$ is defined by (22), by (31) we obtain $\phi_{G}\left(\bar{W}_{i}, \bar{W}_{j}, \eta_{i, j}\right)=\mathbb{F}_{C}\left(\bar{W}_{i}, \bar{W}_{j}, \eta_{i, j}\right)$. Then, (32) is verified by hypothesis (30).

Remark 3 If $\mathcal{A}(\bar{W})$ is non-singular, instead of (31) it is simpler to verify the condition

$$
\mathcal{A}\left(\bar{W}_{i}, \bar{W}_{j}, \eta_{i, j}\right) \frac{\bar{W}_{j}-\bar{W}_{i}}{d_{i j}}=\widehat{G}_{i j}\left(\bar{W}_{i}, \bar{W}_{j}, \eta_{i, j}\right)
$$

Remark 4 Condition (30) means that the discrete flux across the boundary of cell $V_{i}$ balances the discrete source term on $V_{i}$; while condition (31) means that the normal derivative of the discrete flux along the direction $\eta_{i j}$ balances the component of the source term projected into the direction $\eta_{i j}$.

\section{Application to Shallow Water Equations (SWE)}

In this section we apply the previous proposed method to approximate the 2D SWE. These equations describe the behaviour of a flow in a channel, a lake or coastal areas.

The unknowns are

$$
W=\left(h, q_{1}, q_{2}\right)^{T}
$$

where $h$ is the height of the water colum and $q=\left(q_{1}, q_{2}\right)$ the discharge in the $x$ and $y$ directions.

The SWE can be written as a two-dimensional hyperbolic conservation law with source term (14), where the flux functions are

$$
F_{1}(W)=\left(\begin{array}{c}
q_{1} \\
\frac{q_{1}^{2}}{h}+\frac{1}{2} g h^{2} \\
\frac{q_{1} q_{2}}{h}
\end{array}\right), \quad F_{2}(W)=\left(\begin{array}{c}
q_{2} \\
\frac{q_{1} q_{2}}{h} \\
\frac{q_{2}^{2}}{h}+\frac{1}{2} g h^{2}
\end{array}\right) ;
$$

and the source term corresponding to variable bottom is

$$
G(\mathbf{x}, W)=\left(\begin{array}{c}
0 \\
g h \frac{\partial H}{\partial x}(\mathbf{x}) \\
g h \frac{\partial H}{\partial y}(\mathbf{x})
\end{array}\right)
$$


By $H$ we denote the depth of the bottom from a fixed reference level $\bar{A}$ : $H=\bar{A}-z_{b}(\mathbf{x})$, where $z_{b}(\mathbf{x})$ is the bottom function. We also denote $\vec{q}=\left(q_{1}, q_{2}\right)$.

If we also consider friction effects, we have an extra source term $G_{2}$,

$$
G_{2}(W)=g h\left(\begin{array}{c}
0 \\
\vec{S}_{f}
\end{array}\right)
$$

with

$$
\vec{S}_{f}=\vec{q} \frac{\|\vec{q}\|}{h^{2} C^{2} R_{h}}
$$

where $R_{h}$ is the hydraulic ratio, that can be approximated by $h$. For Manning's law we have $C=R_{h}^{1 / 6} / n^{2}$ where $n$ is the bed roughness coefficient.

We can consider $G_{2}$ as a function of $h, q_{1}, q_{2}$ and $\|\vec{q}\|=\sqrt{q_{1}^{2}+q_{2}^{2}}$ : $G_{2}(W)=G_{2}\left(h,\|q\|, q_{1}, q_{2}\right)$. To discretize $G_{2}$, we consider a semi-implicit discretization: If by $L\left(W^{n}\right)$ we denote the spatial discretization of the system without including the term $G_{2}$, we update the solution by solving the linear system

$$
W_{i}^{n+1}=W_{i}^{n}+\Delta t L\left(W^{n}\right)+\Delta t G_{2}\left(h^{n},\left\|\vec{q}^{n}\right\|, q_{1}^{n+1}, q_{2}^{n+1}\right)
$$

So, in what follows, we focus our attention on the discrezation of SWE system without friction terms.

In order to define a family of numerical schemes we begin by considering the homogeneous case, $\phi$ :

$$
\phi\left(W_{i}, W_{j}, \eta_{i j}\right)=\mathbb{F}_{C}\left(W_{i}, W_{j}, \eta_{i j}\right)-\nu_{i j} D\left(W_{i}, W_{j}, \eta_{i j}\right) \frac{W_{j}-W_{i}}{d_{i j}} .
$$

We consider the following definition of $\mathbb{F}_{C}$,

$$
\mathbb{F}_{C}\left(W_{i}, W_{j}, \eta_{i j}\right)=\frac{\mathbb{F}\left(W_{(1-\alpha) i+\alpha j}\right)+\mathbb{F}\left(W_{\alpha i+(1-\alpha) j}\right)}{2} \cdot \eta_{i j},
$$

where we denote $W_{(1-\alpha) i+\alpha j}=(1-\alpha) W_{i}+\alpha W_{j}$ with $\alpha \in[0,1]$.

This expression is used in [13] to avoid entropy corrections in critical points. The authors also propose a definition for the value $\alpha$ as a function depending on the variables. However, the constant value $\alpha=1 / 8$ is shown to provide good results in practically all cases. For the numerical tests that we present in this paper we have set $\alpha=1 / 8$.

As matrix $D$, we can use the absolute value of Roe matrix, corresponding to Roe method, or $D=(\Delta t / \Delta x) A^{2}$, for Lax-Wendroff method. To obtain a stable modification of Lax-Wendroff scheme, we combine both matrices by using flux-limiters. Also, to obtain an extension of Lax-Friedrichs scheme we can use $D=(\Delta x / \Delta t) I$, if by $I$ we denote the identity matrix. 


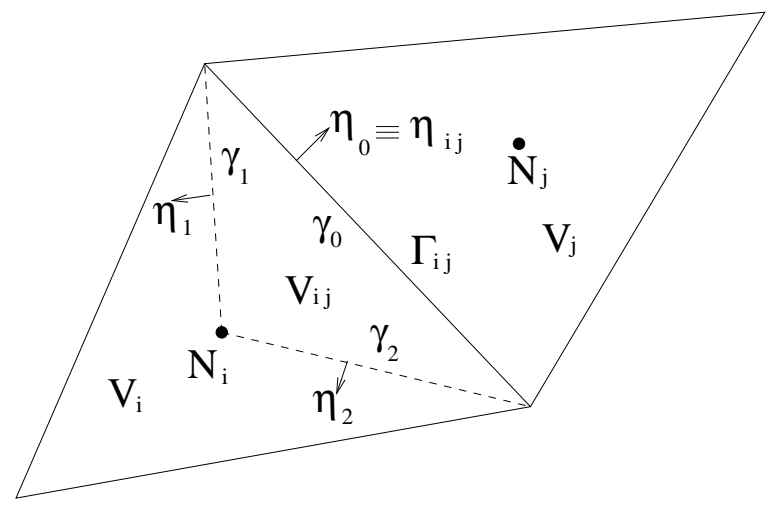

Figure 2: Definition of $V_{i j}$ and $\gamma_{l}$.

Following the previous section, to define the numerical source term (15), it is enough to give $G_{i}$ and $\widehat{G}_{i j}$. To define these two discretizations of $G$, we rewrite the source term (35) as

$$
G(\mathbf{x}, W)=f(W)\left(\begin{array}{c}
0 \\
\partial H / \partial x \\
\partial H / \partial y
\end{array}\right) \text { where } f(W)=g h .
$$

There is a first problem to approximate $G$, as frequently we have not an analytical expression for the bottom function. In what follows, we suppose that we know the values $H_{i}$, approximation of $H\left(N_{i}\right)$ on the control volume $V_{i}$ at the point $N_{i} \in V_{i}$. Therefore, we must set $G_{i}$ and $\widehat{G}_{i j}$ in terms of the values $H_{i}$ and $H_{j}$ with $j \in K_{i}$.

\section{- Definition of $G_{i}$.}

We remark that $G_{i}$ is defined as an approximation of $G$ on the control volume $V_{i}$,

$$
G_{i} \approx \frac{1}{\left|V_{i}\right|} \int_{V_{i}} G(\mathbf{x}, W) \mathrm{d} \mathbf{x}, \quad \text { with } \quad G(\mathbf{x}, W)=f(W)\left(\begin{array}{c}
0 \\
\nabla H
\end{array}\right) .
$$

Let us define $V_{i j}$ as the subset of $V_{i}$ whose boundary is defined by the union of $\Gamma_{i j}$ and the two segments that connect $N_{i}$ with the extremities of $\Gamma_{i j}$ (See Figure 2).

We use the quadrature formula formula

$$
\begin{gathered}
\frac{1}{\left|V_{i}\right|} \int_{V_{i}} f(W) \nabla H(\mathbf{x}) \mathrm{d} \mathbf{x}=\frac{1}{\left|V_{i}\right|} \sum_{j \in K_{i}} \int_{V_{i j}} f(W) \nabla H(\mathbf{x}) \mathrm{d} \mathbf{x} \approx \\
\approx \frac{1}{\left|V_{i}\right|} \sum_{j \in K_{i}} f\left(W_{\rho i+(1-\rho) j}\right) \int_{V_{i j}} \nabla H(\mathbf{x}) \mathrm{d} \mathbf{x} .
\end{gathered}
$$


with $\rho=\frac{1}{2}+\alpha(1-\alpha)$. We observe that this value is an intermediate value between $1 / 2$ (for $\alpha=0$ ) and 3/4 (for $\alpha=1 / 2$ ). This definition can be obtained in terms of the integral of $G$ evaluated at a reconstruction operator:

$$
\frac{1}{\left|V_{i}\right|} \int_{V_{i}} f(W) \nabla H(\mathbf{x}) \mathrm{d} \mathbf{x} \approx \frac{1}{\left|V_{i}\right|} \sum_{j \in K_{i}} \int_{V_{i j}} f\left(P_{i j}(W)\right) \nabla H(\mathbf{x}) \mathrm{d} \mathbf{x}
$$

where $P_{i j}$ is a reconstruction operator, that takes the value $W_{i}$ over $N_{i}$ and the value $\alpha W_{\alpha i+(1-\alpha) j}+(1-\alpha) W_{(1-\alpha) i+\alpha j}$ over $\Gamma_{i j}$.

With the purpose of simplifying the notation we shall suppose that $V_{i j}$ is a triangle, where $\gamma_{0}=\Gamma_{i j}$ is the intersection between $V_{i}$ and $V_{j}$, and where $\gamma_{1}, \gamma_{2}$ are the two interior segments of the boundary of $V_{i j}$ into $V_{i}$ (see Figure 2). By $\eta_{l}$, $l=0,1,2$ we denote the outward unit normal vectors to $\gamma_{l}, l=0,1,2$ respectively (so $\left.\eta_{0}=\eta_{i j}\right)$.

Then, by Green's formula we have

$$
\int_{V_{i j}} \nabla H(\mathbf{x}) \mathrm{d} \mathbf{x}=\sum_{l=0}^{2} \int_{\gamma_{l}} H(\mathbf{x}) \cdot \eta_{l} \mathrm{~d} \mathbf{x} .
$$

We approximate the value of $H(\mathbf{x})$ on $\gamma_{0}=\Gamma_{i j}$ by the mean value of $H_{i}$ and $H_{j}$ : $\left(H_{i}+H_{j}\right) / 2$. On the two interior segments to $V_{i}\left(\gamma_{1}\right.$ and $\left.\gamma_{2}\right)$ we will approximate $H(\mathbf{x})$ by $H_{i}$. Therefore,

$$
\int_{V_{i j}} \nabla H(\mathbf{x}) \mathrm{d} \mathbf{x} \approx \frac{H_{i}+H_{j}}{2}\left\|\gamma_{0}\right\| \eta_{0}+H_{i}\left\|\gamma_{1}\right\| \eta_{1}+H_{i}\left\|\gamma_{2}\right\| \eta_{2}=\frac{\left\|\Gamma_{i j}\right\|}{2}\left(H_{j}-H_{i}\right) \eta_{i j}
$$

To prove the last equality, we have considered that when $H \equiv 1$, we have

$$
0=\int_{V_{i j}} \nabla 1 \mathrm{~d} \mathbf{x}=\sum_{l=0}^{2}\left\|\gamma_{l}\right\|_{l} \eta_{l}
$$

Finally, due to (39)-(40) we define

$$
G_{i}=\frac{1}{\left|V_{i}\right|} \sum_{j \in K_{i}} f\left(W_{\rho i+(1-\rho) j}\right) \frac{\left\|\Gamma_{i j}\right\|}{2}\left(H_{j}-H_{i}\right)\left(\begin{array}{c}
0 \\
\eta_{i j}
\end{array}\right)
$$

with $\rho=\frac{1}{2}+\alpha(1-\alpha)$.

Moreover, if we define $H_{(1-\alpha) i+\alpha j}=(1-\alpha) H_{i}+\alpha H_{j}$, it is easy to see that the previous definition of $G_{i}$ is equivalent to

$$
\begin{aligned}
G_{i}= & \frac{1}{\left|V_{i}\right|} \sum_{j \in K_{i}} \frac{g\left\|\Gamma_{i j}\right\|}{2}\left(\frac{h_{i}+h_{(1-\alpha) i+\alpha j}}{2}\left(H_{(1-\alpha) i+\alpha j}-H_{i}\right)+\right. \\
& \left.+\frac{h_{i}+h_{\alpha i+(1-\alpha) j}}{2}\left(H_{\alpha i+(1-\alpha) j}-H_{i}\right)\right)\left(\begin{array}{c}
0 \\
\eta_{i j}
\end{array}\right) .
\end{aligned}
$$




\section{- Definition of $\widehat{G}_{i j}$.}

To define $\widehat{G}_{i j}$ we remark that, following the construction of the scheme, it is an approximation of $G(R(\mathbf{x}), W(R(\mathbf{x})))$ with

$$
R(\mathbf{x})=\left[\left(\mathbf{x}-P_{i j}\right) \cdot \eta_{i j}\right] \eta_{i j}+P_{i j} \quad \text { where } \quad P_{i j} \in \Gamma_{i j} .
$$

Then, in the case of $G$ defined by (35) we must approximate

$$
G(x, W)=f(W)\left(\begin{array}{c}
0 \\
\nabla H(R(\mathbf{x}))
\end{array}\right), \quad \text { with } \quad f(W)=g h .
$$

If we denote $x_{1}=x$ and $x_{2}=y$, we have

$$
\frac{\partial H(R(\mathbf{x}))}{\partial x_{l}}=\nabla H(R(\mathbf{x})) \cdot \frac{\partial R(\mathbf{x})}{\partial x_{l}}=\frac{\partial H}{\partial \eta_{i j}}(R(\mathbf{x}))\left(\eta_{i j}\right)_{l}, \quad l=1,2 .
$$

And an approximation, of first order of $\frac{\partial H}{\partial \eta_{i j}}(R(\mathbf{x}))$ on $\Gamma_{i j}$ is

$$
\frac{\partial H}{\partial \eta_{i j}}\left(P_{i j}\right) \simeq \frac{H\left(N_{j}\right)-H\left(N_{i}\right)}{d_{i j}}
$$

Then, by (43) and (44) we define

$$
\widehat{G}_{i j}=g \frac{h_{i}+h_{j}}{2} \frac{H_{j}-H_{i}}{d_{i j}}\left(\begin{array}{c}
0 \\
\eta_{i j}
\end{array}\right) .
$$

With these definitions of $\mathbb{F}_{C}, G_{i}$ and $\widehat{G}_{i j}$ we have the following result.

TheOREM 4 Let $D$ be any arbitrary upwinding matrix and $\alpha$ be any arbitrary parameter $\alpha \in[0,1]$. Then, the scheme defined by (15), (22), (38), (42), (45) exactly balances the stationary solution

$$
W=(H, 0,0)^{T}
$$

corresponding to water at rest.

Proof: To prove this property we remark that by Theorem 3 it is sufficient to see that

$$
\frac{1}{\left|V_{i}\right|} \sum_{j \in K_{i}}\left\|\Gamma_{i j}\right\| \mathbb{F}_{C}\left(W_{i}, W_{j}, \eta_{i j}\right)=G_{i},
$$

and

$$
\frac{W_{j}-W_{i}}{d_{i j}}=\widetilde{\mathcal{A}^{-1}}\left(W_{i}, W_{j}, \eta_{i j}\right) \widehat{G}_{i j}
$$


for the stationary solution (46). For this solution we have

$$
\mathbb{F}_{C}\left(W_{i}, W_{j}, \eta_{i j}\right)=\frac{1}{4} g\left(h_{(1-\alpha) i+\alpha j}^{2}+h_{\alpha i+(1-\alpha) j}^{2}\right)\left(\begin{array}{c}
0 \\
\eta_{i j}
\end{array}\right) .
$$

Moreover, by Green's formula, we have

$$
\sum_{j \in K_{i}}\left\|\Gamma_{i j}\right\| \mathbb{F}\left(W_{i}\right) \cdot \eta_{i j}=\mathbb{F}\left(W_{i}\right) \int_{V_{i}} \nabla \cdot\left(\begin{array}{ll}
1 & 0 \\
0 & 1
\end{array}\right) d \mathbf{x}=0
$$

Then,

$$
\begin{gathered}
\frac{1}{\left|V_{i}\right|} \sum_{j \in K_{i}}\left\|\Gamma_{i j}\right\| \mathbb{F}_{C}\left(W_{i}, W_{j}, \eta_{i j}\right)=\frac{1}{\left|V_{i}\right|} \sum_{j \in K_{i}}\left\|\Gamma_{i j}\right\|\left(\mathbb{F}_{C}\left(W_{i}, W_{j}, \eta_{i j}\right)-\mathbb{F}\left(W_{i}\right) \cdot \eta_{i j}\right)= \\
=\frac{1}{\left|V_{i}\right|} \sum_{j \in K_{i}} \frac{g\left\|\Gamma_{i j}\right\|}{4}\left(h_{(1-\alpha) i+\alpha j}^{2}-h_{i}^{2}+h_{\alpha i+(1-\alpha) j}^{2}-h_{i}^{2}\right)\left(\begin{array}{c}
0 \\
\eta_{i j}
\end{array}\right) .
\end{gathered}
$$

As for the stationary solution defined by (46) we have $h=H$, and $G_{i}$ is defined by (42), we obtain that $G_{i}$ coincides with (49). Then, (47) is verified.

Next, we must prove that (48) is verified for the stationary solution (46). But for this stationary solution matrix $\mathcal{A}$ is non singular. Then,

$$
\widetilde{\mathcal{A}^{-1}}\left(W_{i}, W_{j}, \eta_{i j}\right)=\mathcal{A}^{-1}\left(\frac{W_{i}+W_{j}}{2}, \eta_{i j}\right) \text {. }
$$

Then, it is enough to check that

$$
\mathcal{A}\left(\frac{W_{i}+W_{j}}{2}, \eta_{i j}\right) \frac{W_{j}-W_{i}}{d_{i j}}=\widehat{G}_{i j}
$$

and

$$
\mathcal{A}\left(\frac{W_{i}+W_{j}}{2}, \eta_{i j}\right)=\left(\begin{array}{ccc}
0 & \eta_{i j, 1} & \eta_{i j, 2} \\
g h_{(i+j) / 2} \eta_{i j, 1} & 0 & 0 \\
g h_{(i+j) / 2} \eta_{i j, 2} & 0 & 0
\end{array}\right)
$$

Finally, as $h=H$ for this stationary solution, $\widehat{G}_{i j}$ defined by (45) coincides with

$$
\mathcal{A}\left(\frac{W_{i}+W_{j}}{2}, \eta_{i j}\right) \frac{W_{j}-W_{i}}{d_{i j}}=g \frac{h_{j}+h_{i}}{2} \frac{h_{j}-h_{i}}{d_{i j}}\left(\begin{array}{c}
0 \\
\eta_{i j}
\end{array}\right)
$$

what concludes the proof. 


\subsection{Numerical treatment of wet/dry fronts}

Besides the difficulties related to source terms, some specific problems appear in the particular case of SWE, in particular the numerical simulation of wet/dry fronts that appear when the thickness of the water layer vanishes. The numerical treatment of this front is important in emerging bottom topographies because the gradient of the bottom function $H$ generates spurious pressure forces that can make the fluid to claim up steps or slopes in a non-physical way. This treatment can also directly influence the velocity of the front. The treatment of wet/dry fronts is also related to the balance properties of the method.

We use here the numerical treatment proposed by Castro et al. in [7]. It basically consists in a local redefinition of the topography in the inter-cells corresponding to wet/dry transitions in order to avoid the generation of spurious pressure forces. Another wet/dry treatment is presented in [8] considering the Godunov flux in the inter-cell corresponding to wet/dry transition. A nonlinear Riemann problem whose solution depends on the bottom jump and the fluid regime is solved.

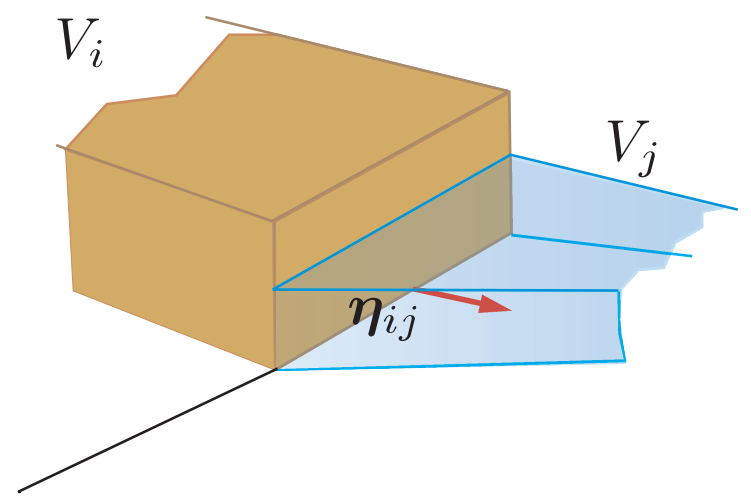

(a) Case 1

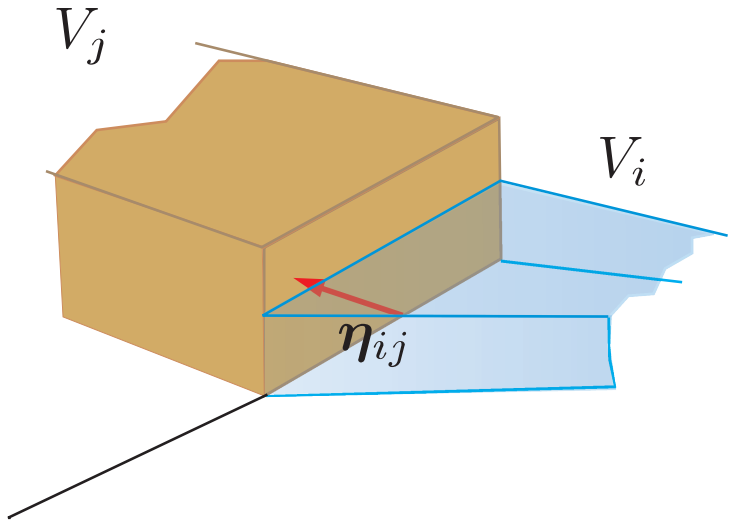

(b) Case 2

Figure 3: Emerging bottom configurations

In order to apply the numerical technique proposed in [7], first, we note that the numerical scheme (15), (22), (38), (41), (45), can be written under the following structure:

$$
W_{i}^{n+1}=W_{i}^{n}-\frac{\Delta t}{\left|V_{i}\right|} \sum_{j \in K_{i}}\left\|\Gamma_{i j}\right\| \mathcal{F}_{G, i j}^{-},
$$

where

$$
\begin{aligned}
& \mathcal{F}_{G, i j}^{-}=\frac{\mathbb{F}\left(W_{(1-\alpha) i+\alpha j}\right)+\mathbb{F}\left(W_{\alpha i+(1-\alpha) j}\right)}{2} \cdot \eta_{i j}-\frac{1}{2} D\left(W_{i}, W_{j}, \eta_{i j}\right)\left(W_{j}-W_{i}\right)+ \\
& +\left(\frac{1}{2} g h_{\rho i+(1-\rho) j}-\frac{1}{2} D\left(W_{i}, W_{j}, \eta_{i j}\right) \widetilde{A^{-1}}\left(W_{i}, W_{j}, \eta_{i j}\right) g h_{(i+j) / 2}\right)\left(H_{j}-H_{i}\right)\left(\begin{array}{c}
0 \\
\eta_{i j}
\end{array}\right),
\end{aligned}
$$

with $\rho=\frac{1}{2}+\alpha(1-\alpha)$. 
The numerical treatment of wet/dry fronts used in this work can be summarized as follows: Let us suppose that an emerging bottom situation as the one shown in Figure 3(a) arises at the edge $\Gamma_{i j}$, where $h\left(N_{i}\right)=0$ and $h\left(N_{j}\right) \leq H\left(N_{j}\right)-H\left(N_{i}\right)$. In this case, instead of using $(50), \mathcal{F}_{G, i j}^{-}$is given by:

$$
\mathcal{F}_{G, i j}^{-}=(0,0,0)^{T} \text {. }
$$

Conversely, in the situation shown in Figure 3(b), where $h\left(N_{i}\right) \leq H\left(N_{i}\right)-H\left(N_{j}\right)$ and $h\left(N_{j}\right)=0, \mathcal{F}_{G, i j}^{-}$is given by:

$$
\mathcal{F}_{G, i j}^{-}=\frac{\mathbb{F}\left(W_{(1-\alpha) i+\alpha j}\right)+\mathbb{F}\left(W_{\alpha i+(1-\alpha) j}\right)}{2} \cdot \eta_{i j}-\frac{1}{2} g \rho h_{i}^{2}\left(\begin{array}{c}
0 \\
\eta_{i j}
\end{array}\right) .
$$

This modification can be interpreted as follows: at the edges where emerging bottom situations arise, the projected Riemann Problem is substituted by an approximate partial Riemann problem (see [8] for details).

Some straightforward calculations show that Theorem 4 is also verified for water at rest when there are wet/dry areas.

\section{$6 \quad$ Numerical tests}

In this section we present three numerical tests. In the first one, we consider a one-dimensional flux that consists in a double supercritical rarefaction wave, where one of them finds an obstacle and a shock is produced. We compare in this test the solution obtained with the 2D solver using a non-structured mesh with the numerical results for the $1 \mathrm{D}$ solver. In Test 2 we present the evolution in time until a stationary solution that presents a jump and a drop. A turbulence viscosity term is included in the system, following [31]. We follow Roache's grid convergence criterion, to ensure the grid convergence to the stationary solution. Finally, in Test 3, we study the ecological disaster of Aznalcóllar's dam break. In this case we test the arrival time of the flow to a seating capacity station and the limits of the flood.

For tests 1 and 3 we consider finite control volumes of type edge (also called of type baricentric). This type of control volume is defined as follows: We consider an edge $E$ of the triangulation $\mathcal{T}_{h}$, then the control volume $V_{E}$ is the subset that contains the fixed edge $E$ and whose boundary is defined by the union of the segments that connect the baricenters of the two triangles that contain $E$ and the extremities of the edge (see Figure 4).

We consider the numerical scheme defined by (15)-(22) with $\alpha=1 / 8$ and

$$
D\left(W_{i}, W_{j}, \eta_{i j}\right)=\left|\mathcal{A}\left(\left(W_{i}+W_{j}\right) / 2, \eta_{i j}\right)\right| .
$$

As CFL condition we use $c f l=0.8$. This condition, for the two-dimensional system, is imposed in such a way that $\Delta t$ verifies

$$
\frac{\Delta t}{\left|V_{i}\right|} \sum_{j \in K_{i}}\left\|\Gamma_{i j}\right\| \max _{l}\left\{\left|\lambda_{i j, l}\right|\right\} \leq 2 c f l \quad \forall i
$$




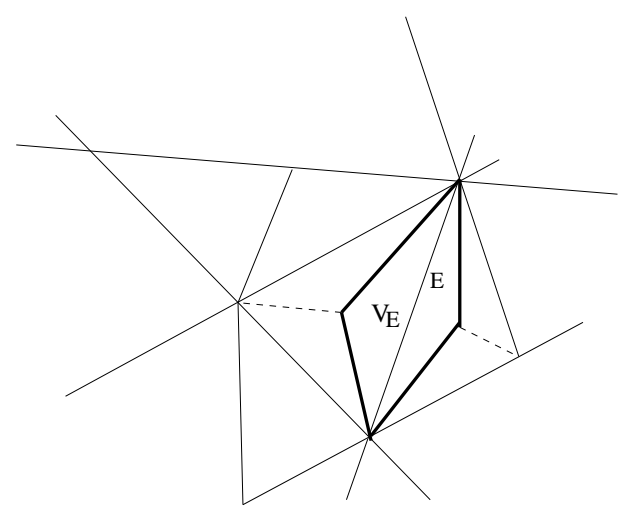

Figure 4: Finite volume mesh of edge type

where by $\lambda_{i j, l} l=1, \ldots, N$ we denote the eigenvalues of $\mathcal{A}\left(\left(W_{i}+W_{j}\right) / 2, \eta_{i j}\right)$.

\subsection{A double rarefaction wave with vacuum}

In this subsection we present a test proposed by Galloüet in [16]. The initial conditions are

$$
h(\mathbf{x}, 0)=H(\mathbf{x}), \quad q(\mathbf{x}, 0)= \begin{cases}350 & \text { if } x>50 / 3 \\ -350 & \text { if } x<50 / 3\end{cases}
$$

where $H(\mathbf{x})=10-z_{b}(\mathbf{x})$, and the bottom function is defined by

$$
z_{b}(\mathbf{x})=z_{b}(x, y)= \begin{cases}1 & \text { if } 25 / 3<x<25 / 2 \\ 0 & \text { otherwise }\end{cases}
$$

This is a one-dimensional test initially proposed by Toro (see [30]) over a flat bottom. In [16] the authors propose to introduce a bump on the bottom, on the left of $x=50 / 3$.

We have a supercritical flow as initial conditions, that is separated at the left and the right of $x=50 / 3$, with Froude numbers $\pm 3,533$. The test consists in two supercritical waves which instantly separate the water column at $x=50 / 3$ and produce a vacuum. The wave traveling to the left presents a shock in the water surface, produced by the presence of the bump. The purpose of this test is to study the robustness of the numerical schemes, including the numerical treatment of wet/dry fronts (see Section 5.1).

We compare the solution obtained with the $1 \mathrm{D}$ solver with the result of the $2 \mathrm{D}$ solver using a non-structured mesh. The domain of the $1 \mathrm{D}$ problem is $[0,25]$ and for the $2 \mathrm{D}$ we consider $[0,25] \times[0,1]$. The final time is fixed at $t=0.25 \mathrm{~s}$.

At $y=0$ and $y=1$ we impose as boundary condition $q \cdot \eta=0$ as it was proposed in [4]. At $x=0$ and $x=25$ we duplicate the states, for the virtual control volumes adjacent to the boundary and exterior to the domain (See Section 3). 


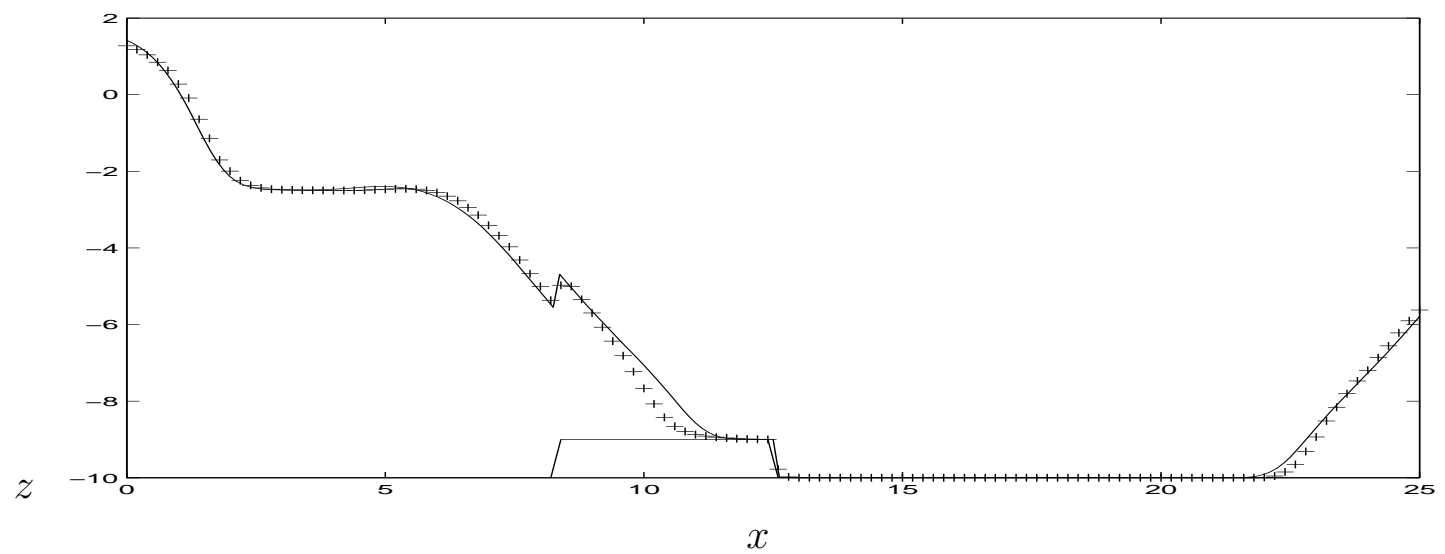

Figure 5: Analysis of the 1D character of the 2D solution. Water surface $(h-H)$ on $y=0.5$ (cross-line) and one-dimensional water surface solution (continuous line).

For the $1 \mathrm{D}$ problem we consider $\Delta x=0.25$. In the case of the $2 \mathrm{D}$ problem, we have used finite control volumes of type edge which are built by a non-structured mesh with 3960 triangles.

In Figure 5 we compare the results of water surface $(h-H)$ of the two-dimensional scheme at $y=0.5$ with the ones of the $1 \mathrm{D}$ scheme. In Figure 6 we compare the water surface provided by the $2 \mathrm{D}$ scheme at $y=0$ and $y=0.5$. The main differences occur at the transition of wet/dry areas on the obstacle. This may be due to the loss of accuracy produced by the representation of the sharp sides of the obstacle in the non-structured grid.

We also observe that $1 \mathrm{D}$ and $2 \mathrm{D}$ scheme provide close solutions, where there is no obstacle, the transition of wet/dry areas is well captured, and shocks and rarefaction waves are also well captured.

We finally observe in Figure 6 that the 1D nature of the solution is quite well represented by our solver, again with some disagreements near sharp bottom gradients.

\subsection{Hydraulic jump and hydraulic drop}

In this subsection we present a test proposed in [31], where an hydraulic jump and an hydraulic drop are formed.

To study the formation of hydraulic jumps in open channel flows Zhou and Stansby propose in [31] a stationary system based in SWE with friction and a turbulence viscosity term. We consider the unsteady system and study the convergence in time towards the stationary solution. The system proposed in [31] can be written 

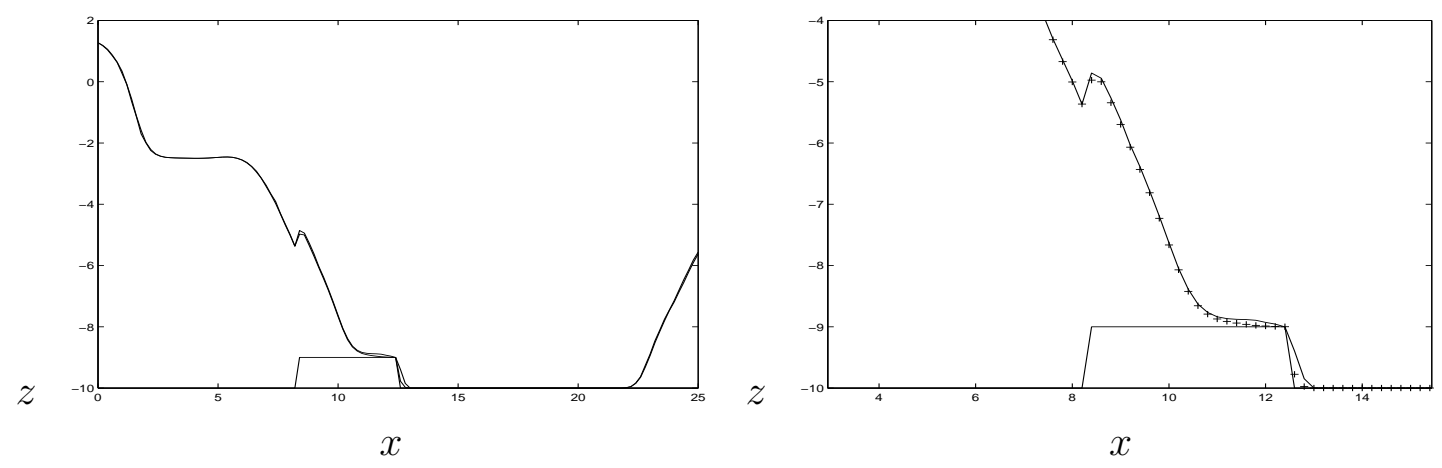

Figure 6: Analysis of the 1D character of the 2D solution. Left: Water surface at $y=0$ and $y=0.5$. Right: Zoom at the bottom step $(y=0.5$ cross-line, $y=0$ continous line)

as follows

$$
\frac{\partial W}{\partial t}+\partial_{x} F_{1}(W)+\partial_{y} F_{2}(W)=G(W)+G_{2}(W)+\operatorname{div}\left(h \nu_{t}(W) \nabla\left(\begin{array}{c}
0 \\
\vec{U}
\end{array}\right)\right)
$$

where $W$ is the vector of unknowns $(33),\left(F_{1}, F_{2}\right)=\mathbb{F}$ is defined by $(34), G$ is the source term due to the topography defined by (35) and $G_{2}$ is the source term of friction terms defined by (36). We denote $\vec{U}=\left(q_{1} / h, q_{2} / h\right)$. Finally, the turbulent viscosity coefficient $\nu_{t}(W)$ is defined by (see [31]) $\nu_{t}(W)=k q_{*} / 6$, where $k$ is the Von Kármán constant (equal to 0.4 ) and $q_{*}=h u_{*}$ where $u_{*}$ is the shear velocity,

$$
q_{*}=\frac{n}{h^{7 / 6}} \sqrt{g\left(q_{1}^{2}+q_{2}^{2}\right)} .
$$

We remember that by $n$ we denote the Manning coefficient (see equation (36)).

To discretize system (52) we consider the proposed discretization defined in Section 5, including a centered discretization of the turbulent viscosity term. Concretely, we consider the scheme

$$
\begin{gathered}
\left|V_{i}\right| \frac{W_{i}^{n+1}-W_{i}^{n}}{\Delta t}+\sum_{j \in K_{i}}\left\|\Gamma_{i j}\right\| \phi_{G}\left(W_{i}^{n}, W_{j}^{n}, \eta_{i j}\right)=\left|V_{i}\right| G_{i}^{n}+ \\
\quad+\sum_{j \in K_{i}}\left\|\Gamma_{i j}\right\| \frac{h_{(i+j) / 2}^{n} \nu_{t,(i+j) / 2}^{n}}{d_{i j}}\left(\begin{array}{c}
0 \\
\vec{U}_{j}^{n}-\vec{U}_{i}^{n}
\end{array}\right)
\end{gathered}
$$

We have denoted $\nu_{t,(i+j) / 2}^{n}=\nu_{t}\left(W_{(i+j) / 2}^{n}\right)$; and $W_{(i+j) / 2}=\left(W_{i}+W_{j}\right) / 2$.

The test is the following. We consider a domain of $30.5 \mathrm{~m}$. length and $1.4 \mathrm{~m}$. width. $H(\mathbf{x})=-z_{b}(\mathbf{x})$, where the bottom function is

$$
z_{b}(\mathbf{x})=z_{b}(x, y)= \begin{cases}0 & \text { if } x<14.5 \\ -0.03(x-14.5) & \text { if } x \geq 14.5\end{cases}
$$


The domain is a rectangular channel with two slopes. An upstream horizontal reach is connected with a reach with a negative slope of -0.03 (See Figure 7).

As initial condition we consider $h=0, \vec{q}=0$ on all the domain. And we introduce a supercritical boundary condition $(F r=4.65)$ at $x=0$,

$$
h(t,(0, y))=0.06, \quad q_{1}(t,(0, y))=0.21426, \quad q_{2}(t,(0, y))=0
$$

We consider a regular rectangular mesh defined by 61 points on edge $x$ and 10 points on edge $y$.

In Figure 7 we present the evolution of the central depth profile at different times: $3,5,10,15,17,30$ and $300 \mathrm{~s}$. We observe the evolution of the water surface until the stationary solution is reached. In Figure 8 we present the evolution of the discharge and a zoom of the discharge at $t=300 \mathrm{~s}$.

The result of this test is a stationary solution where an hydraulic jump appears in the flat reach and a drop appears around the line where the slope of the channel changes.

The jump produces an increase of the water surface level and the flow changes from supercritical to subcritical. We denote by $h_{1}$ the depth on the left and by $h_{2}$ the depth on the right of the jump. Numerically we obtain $h_{1}=0.1137$ and $h_{2}=0.2285$. As we have a stationary jump, by the Rankine-Hugoniot condition or by Bélanger formula (see [31]) we have

$$
\frac{h_{2}}{h_{1}}=\frac{\sqrt{8 F r_{1}^{2}+1}-1}{2}
$$

where $F r_{1}$ is the Froude number at the left of the jump, we obtain $F r_{1}=1.784$. By introducing these values of $h_{1}$ and $F r_{1}$ in (54) we deduce that the value after the jump must be $h_{2}=0.2356$.

In the drop, there is a decrease of water depth, the flow changes from subcritical to supercritical and there is a line of critical points where the slope of the channel changes. The theoretical value of the critical depth $\left(h_{c}\right)$ at these points must verify that the Froude number is equal to one, $q /\left(h_{c} \sqrt{g h_{c}}\right)=1$, then $h_{c}=0.1673$. Numerically we obtain $h=0.16707$.

The depth profiles that this stationary solution presents, from the left to the right, are: H3, Jump, H2, Drop, S2 and finally an uniform flow (See Figure 7). If we denote by $S_{0}=\partial_{x} H$, we have: at $\mathrm{H} 3 S_{0}=0, F r>1, h<h_{c}$, for $\mathrm{H} 2 S_{0}=0$, $F r<1, h>h_{c}$. In $S_{2}$ we have $S_{0}>S_{c}, F r>1, h_{n}<h<h_{c}$, where $S_{c}=S_{f}\left(h_{c}\right)$, with $S_{f}$ defined by the first component of (37), and $h_{n}$ is the depth for an uniform flow. The uniform flow is obtained when $S_{0}=S_{f}$. For this test we can deduce that the value of the depth for the uniform flow is $h_{n}=0.1054$, numerically we obtain $h=0.1055$.

Finally, we include a grid convergence study. We consider three meshes obtained by halving the grid size. The $x$-space steps are $\Delta x=0.5, \Delta x=0.25$ and $\Delta x=0.125$.

We respectively denote by $W_{1}, W_{2}$ and $W_{3}$ the solutions obtained with the finer mesh, the intermediate mesh and $W_{3}$ the coarser mesh. 

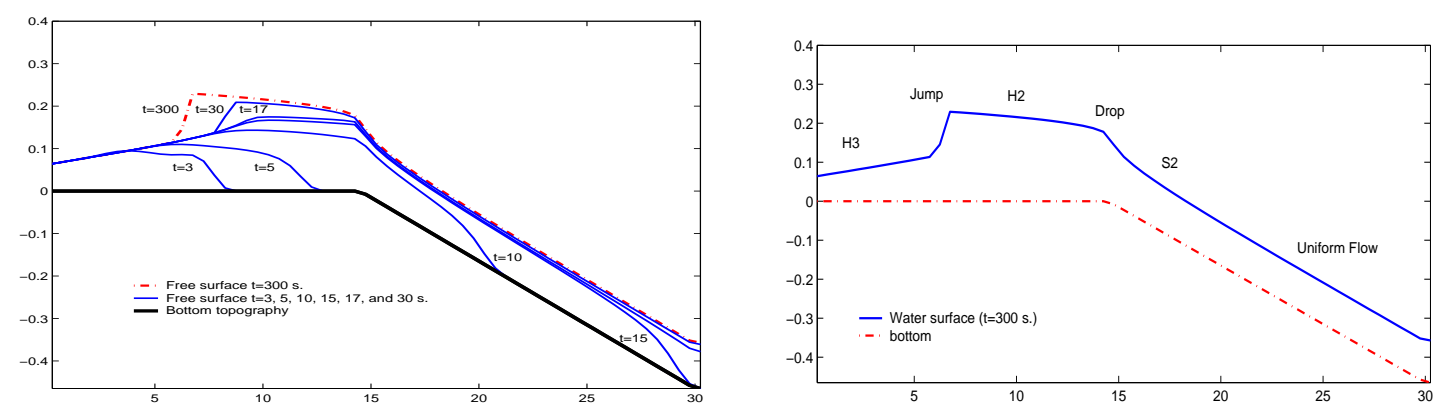

Figure 7: Hydraulic jump and hydraulic drop. Evolution of the water surface. Convergence to the stationary solution. (Profiles of the central depth).

Roache [26] defined an error estimate that contains a safety factor, referred as the Grid Convergence Index or GCI. The GCI gives a band of error around the fine mesh solution. A small value of GCI indicates that the computation is near the asymptotic numerical solution. The GCI factor is defined by

$$
\mathrm{GCI}_{i j}=\frac{F_{s}}{r^{p}-1}\left\|\frac{W_{j}-W_{i}}{W_{i}}\right\| 100 \%
$$

where the safety factor is set to $F_{s}=1.25$. By $r$ we denote the grid refinement factor, $r=2$ for the considered meshes. Using these meshes we compute $\mathrm{GCI}_{12}$ and $\mathrm{GCI}_{23}$. By Theorem 2 we have that the solution studied in this test is preserved up to second order, although the presence of the jump can reduce the order of accuracy. We determine the effective order of convergence for this solution using the solutions in the three meshes, taking as reference solution $W_{1}$, corresponding to the finer mesh. Then, the value of $p$ can be calculated from

$$
p=\frac{\ln \left(\left\|W_{3}-W_{1}\right\|_{L^{1}} /\left\|W_{2}-W_{1}\right\|_{L^{1}}\right)}{\ln (r)} .
$$

We obtain

$$
\left\|W_{3}-W_{1}\right\|_{L^{1}}=0.0776, \quad\left\|W_{2}-W_{1}\right\|_{L^{1}}=0.0246 .
$$

then, using (55) we obtain $p=1.6796$. We also obtain

$$
\left\|\frac{W_{3}-W_{2}}{W_{2}}\right\|_{L^{1}}=0.0343, \quad\left\|\frac{W_{2}-W_{1}}{W_{1}}\right\|_{L^{1}}=0.0092 .
$$

We can now calculate the values of $\mathrm{GCI}_{12}$ and $\mathrm{GCI}_{23}$, we obtain

$$
\mathrm{GCI}_{23}=1.9458 \%, \quad \mathrm{GCI}_{12}=0.5219 \%
$$



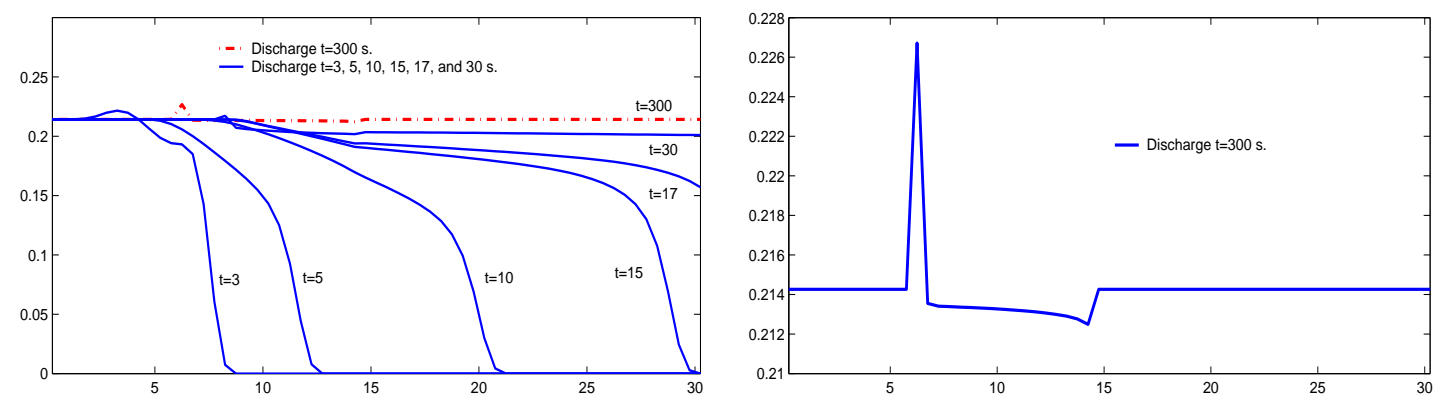

Figure 8: Hydraulic jump and hydraulic drop. Discharge. Left: evolution for $\mathrm{t}=2$, $5,10,15,17,30$ and $300 \mathrm{~s}$. Right: Zoom of the discharge at t=300 s.

We have verified that all grid levels are in the asymptotic range of convergence for the computed solutions. This is checked by observing wether the following relation is verified

$$
\mathrm{GCI}_{23}=r^{p} \mathrm{GCI}_{12} \text {. }
$$

We obtain

$$
\frac{\mathrm{GCI}_{23}}{r^{p} \mathrm{GCI}_{12}}=1.16
$$

\subsection{Numerical simulation of a real dambreak: Aznalcóllar (Spain, 1998)}

In this section we present the results obtained in the simulations of the Aznalcóllar dam break. Aznalcóllar mines are located near Seville (Spain). Residuals produced by the mine activity were stored in two reservoirs located near Guadiamar river.

On April, 25th 1998, a part of the lateral wall of the bigger reservoir was collapsed, provoking a first flood through the Guadiamar basin. After approximately 5 hours and 10 minutes, a part of the wall separating the two reservoirs collapsed, provoking a second flood. An aerial view of the affected zone is shown in Figure 10.

In order to perform the simulation of the flood, first a 2D triangular mesh of the region containing the flooding area is constructed. This mesh is finer in the abrupt zones and, in particular, in the dam area (see Figure 9(a)).

Next, a description of the topography is needed over the mesh, that is, the values of $H(\mathbf{x})$ at all vertices of the mesh must be provided. We have computed them from topographical data provided by Dr. F.J. Segovia Espiau (University of Sevilla) using the procedure described in Appendix A from a CAD format map where the level curves are given (see Figure 9(b)).

Finally, an edge-based finite volume mesh is built using the triangular mesh previously generated, following the procedure described in Appendix A. The final 

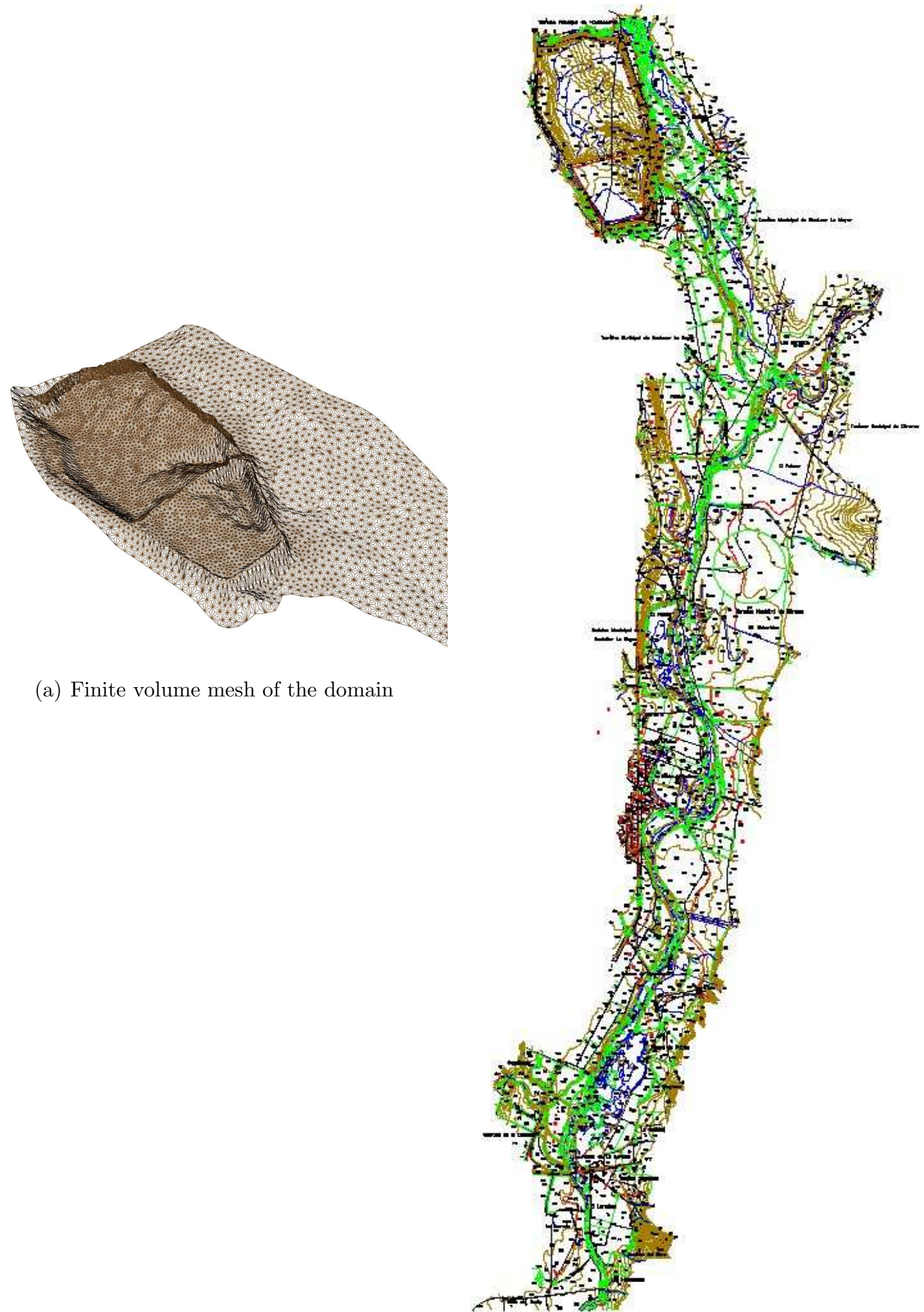

(a) Finite volume mesh of the domain
(b) Topographic map: level curves

Figure 9: Topographic map and finite volume mesh. 


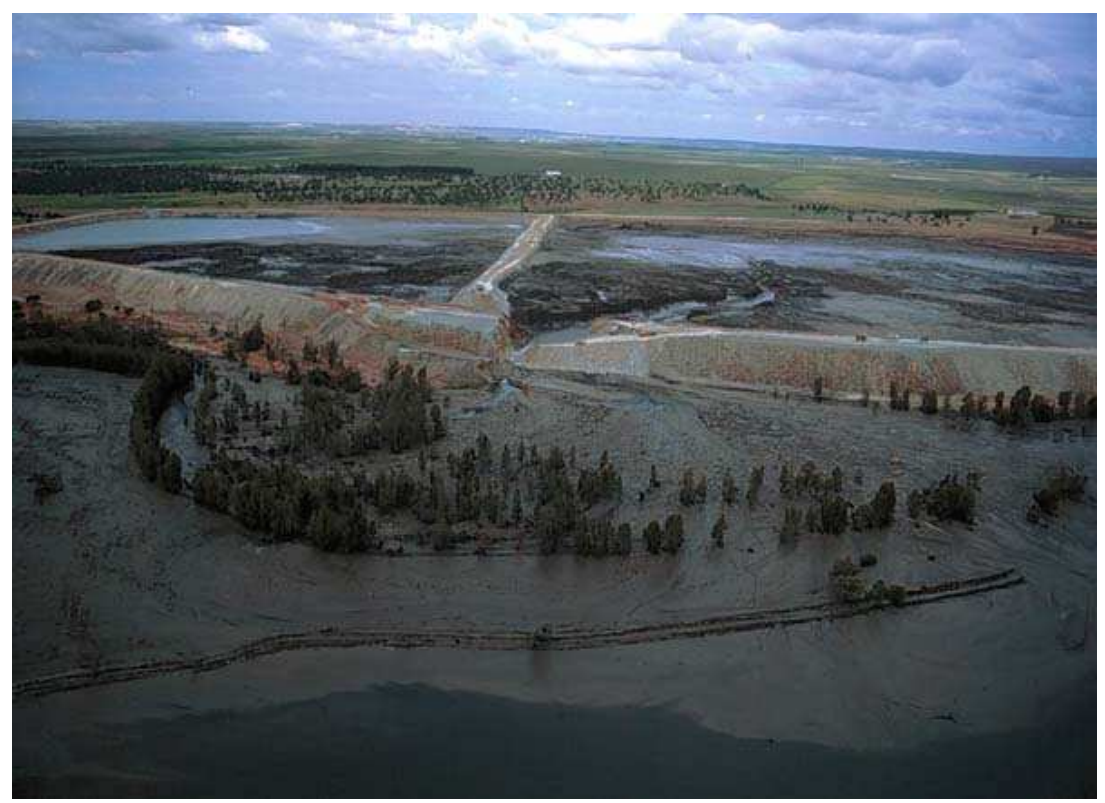

Figure 10: Image after the dam break

mesh is formed by 30398 finite volume cells.

As initial condition, water at rest in the two reservoirs is considered, placing the free surface $30 \mathrm{~cm}$ below the top of the dam walls. In order to simulate the dam break of the first reservoir, a similar hole to the one produced by the wall collapse is considered in the original topography. The collapse of the second reservoir is simulated in the same way.

Just after the dam break two effects take place: A large upstream rarefaction wave and a strong downstream transcritical shock, in the presence of wet/dry transitions and very strong topography gradients. Both are remarkably computed by our solver, that appears to produce the necessary entropy levels.

Some validation of the simulation is possible: the velocity of the flood is checked by comparing the arrival time of the simulated flood with the real one at a seating capacity station located in the basin of the river Guadiamar, practically in the middle of the considered region. The real time that took the flood to arrive to this point, was one hour and forty minutes, while in the simulation time differs less than two minutes (See Figure 17(c)). The Manning coefficient is chosen to $n=0.039$. It is defined as a mean value of the Manning coefficients corresponding to the area where flood is produced. This value fits the flood velocity.

The extension of the numerical flood is compared with real limits in Figure 12(a). An excellent fit takes place in the areas with larger slopes, where both limits practically coincide. In the areas with small slopes, the error is larger. This error possibly arises because sedimentation effects were no considered in this model.

In Figures 11(a) and 11(b) we present two details of the dam area after the dam 
break. In Figure 11(c) a zoom of the dam area at the moment of the collapse of the second reservoir is shown. In Figure 11(d) we present a view of all the domain two minutes after the second reservoir collapses.

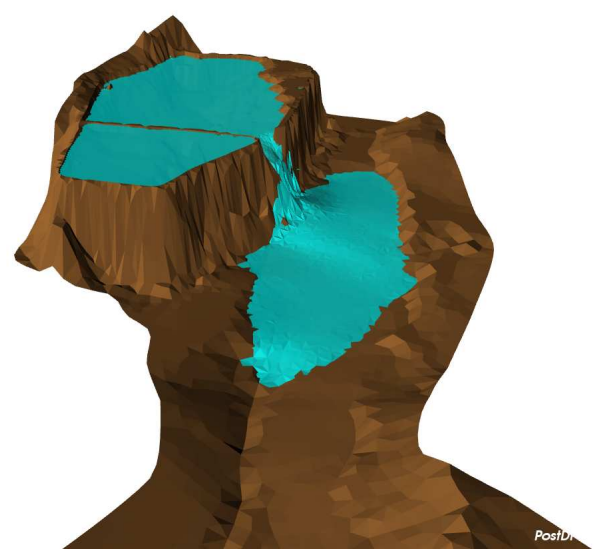

(a) Zoom after the dam break

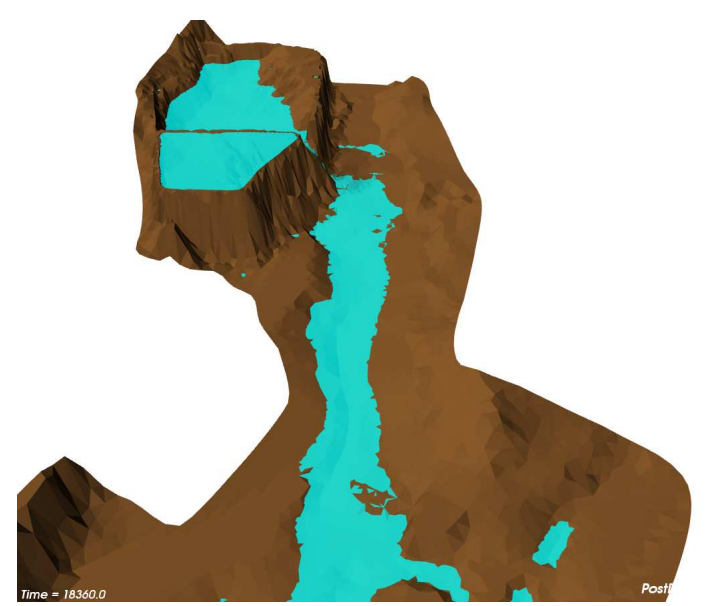

(c) Zoom of the dam area when the second reservoir collapses

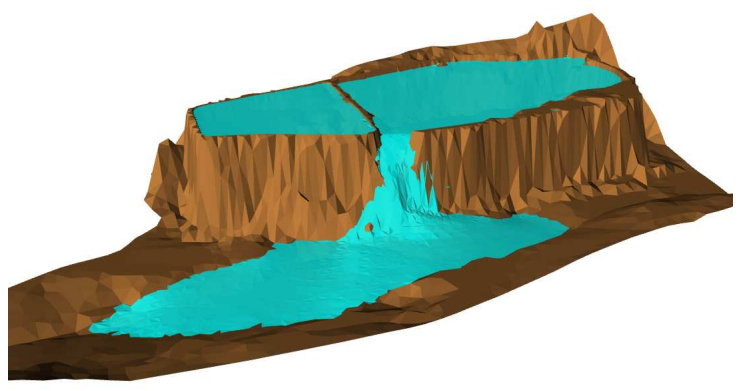

(b) Zoom after the dam break (lateral view)

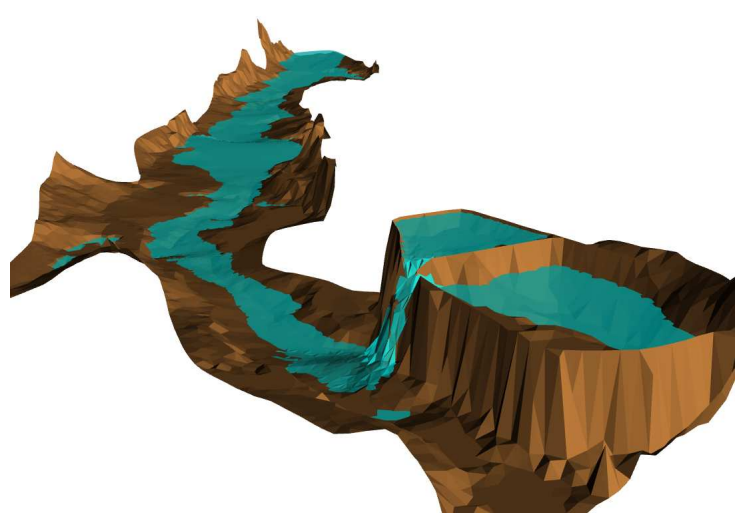

(d) View of all the domain after 2 minutes the second reservoir collapses

Figure 11: Evolution of the flood (Aznalcóllar dam break)

Finally, we show the results corresponding to four sections: Section 1 placed inside the dam; a downstream section (Section 2), a section located on the seating capacity station (Section 3) and a section located downstream the seating capacity station (Section 4) (see Figure 12(b)).

In Figures 13, 14, 15 and 16, the water depth is shown at different times on Sections 1, 2, 3 and 4 respectively.

In Figure 17 several time series showing the depth at selected points chosen in each section (see Figure 12(b)) are depicted. 

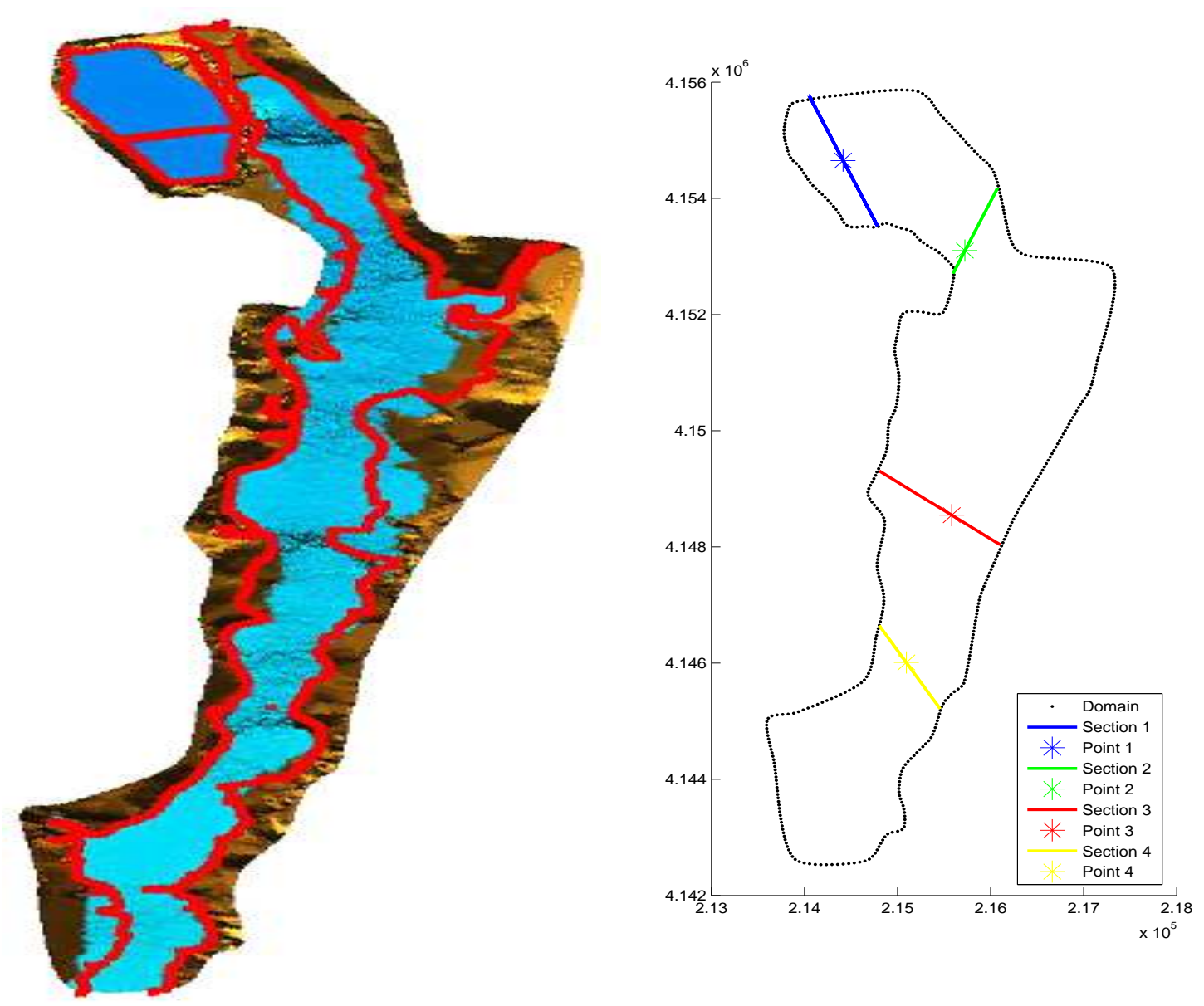

(a) Comparison of real and computed limits of the (b) Selected sections in the domain (scales in meflood ters)

Figure 12: Left: comparison of the real and computed limits. Right: Selected sections in the domain 


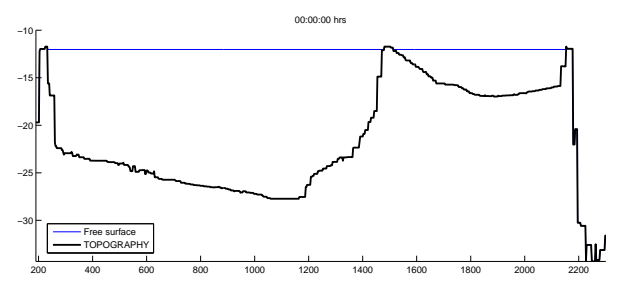

(a) Time $t=0$

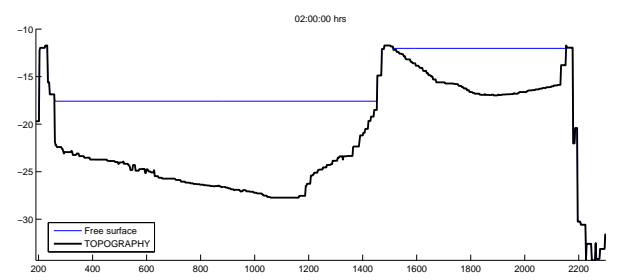

(c) Time $t=2$ hours

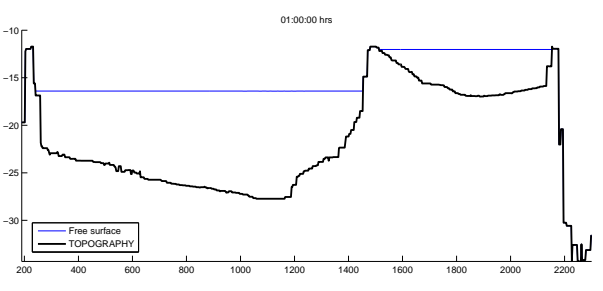

(b) Time $t=1$ hour

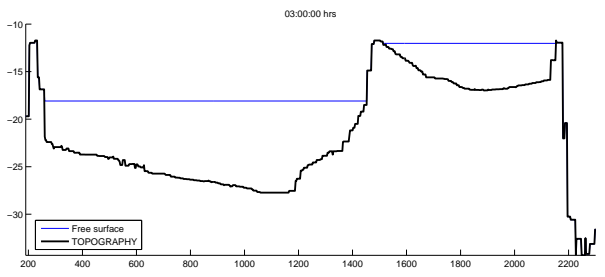

(d) Time $t=3$ hours

Figure 13: Water depths in Section 1

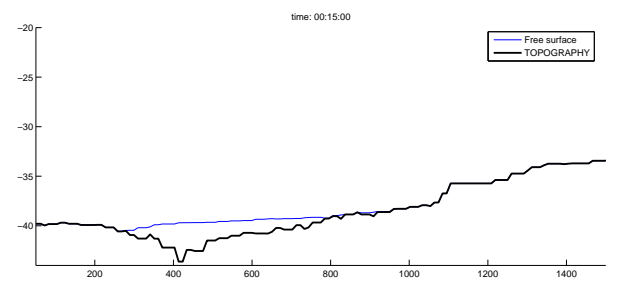

(a) Time $t=15$ minutes

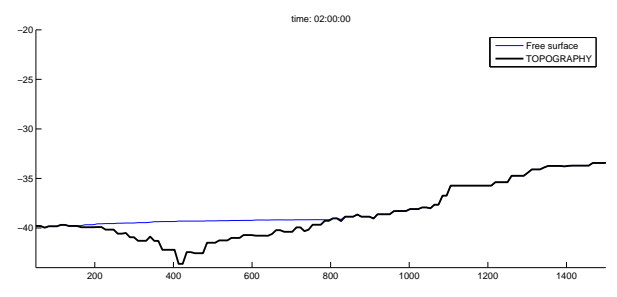

(c) Time $t=2$ hours

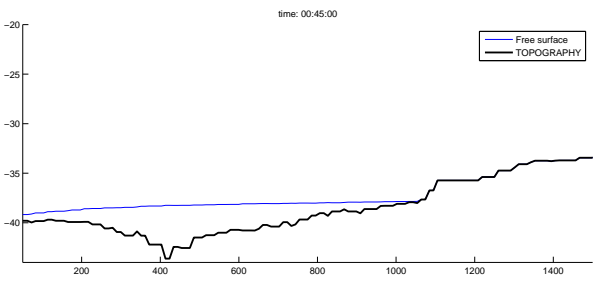

(b) Time $t=45$ minutes

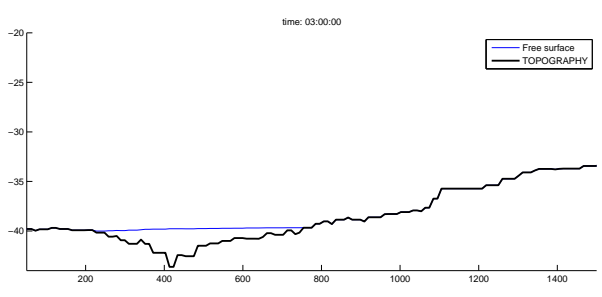

(d) Time $t=3$ hours

Figure 14: Water depths in Section 2 


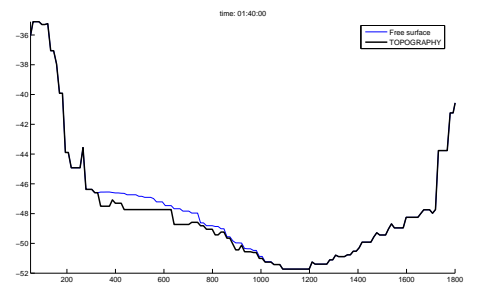

(a) Time $\mathrm{t}=1$ hour and 40 minutes

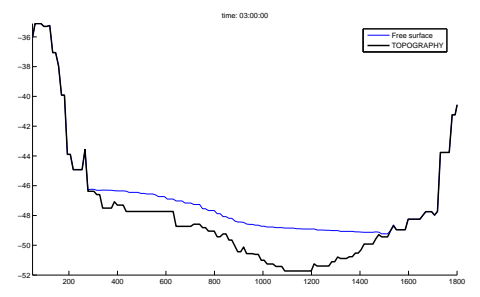

(c) Time $t=3$ hours

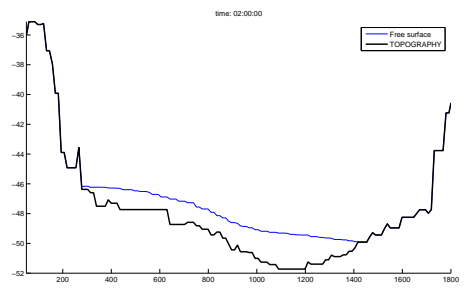

(b) Time $t=2$ hours

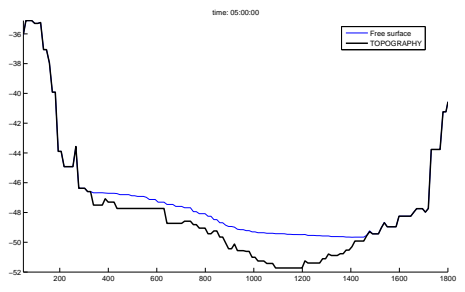

(d) Time $t=5$ hours

Figure 15: Water depths in Section 3

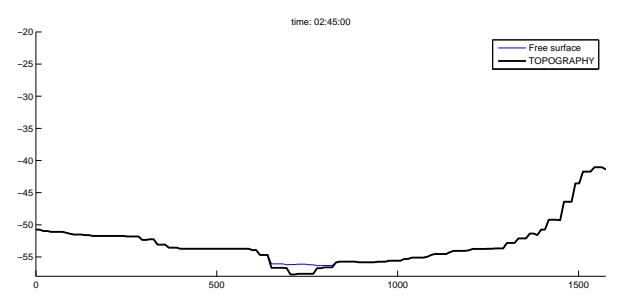

(a) Time $\mathrm{t}=30$ minutes

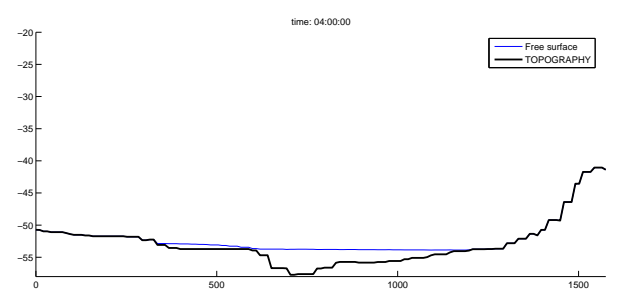

(c) Time $\mathrm{t}=2$ hours

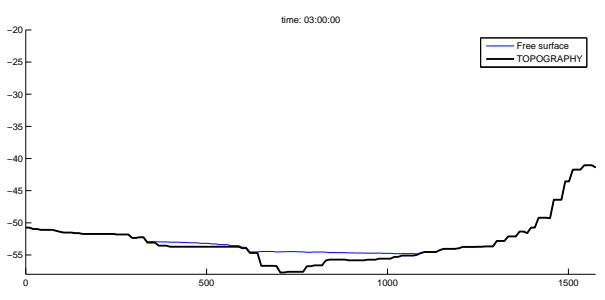

(b) Time $\mathrm{t}=45$ minutes

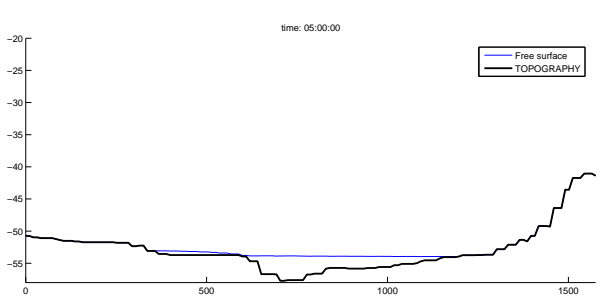

(d) Time $t=3$ hours

Figure 16: Water depths in Section 4 


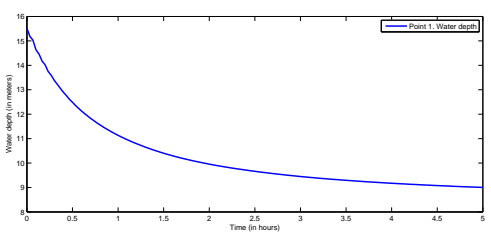

(a) Time series for a point located in Section 1

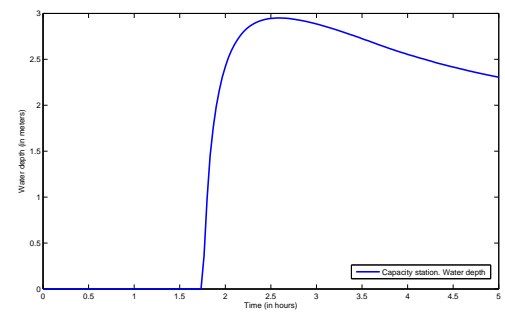

(c) Time series for a point located in Section 3

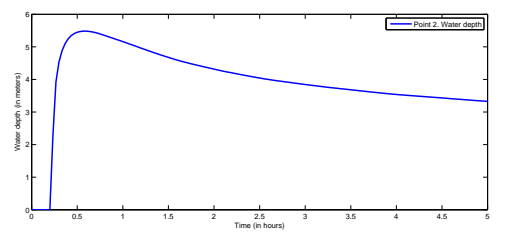

(b) Time series for a point located in Section 2

Figure 17: Depth time series for several points.

\section{Appendix A. Construction of the mesh and inter- polated topography for Aznalcóllar toxic waste test}

We present in this appendix the form in which we have constructed the mesh of triangles to build the finite volume mesh used in the simulation of Subsection 6.3, and the topography.

The objective is to associate a value of the topography to each node of a mesh of triangles of the domain.

We describe the process in three steps.

- Step 1: We build an auxiliar triangulation $\mathcal{T}^{\prime}$. The vertex of the triangles of $\mathcal{T}^{\prime}$ are the points at which we know the topography.

- Step 2: We build a mesh of triangles $\mathcal{T}$ by using adaptive techniques, in order to obtain a finer mesh in areas with larger gradient and near equilateral triangles.

- Step 3: Finally, we interpolate the values of the topography at each node of the triangles of $\mathcal{T}$, with the help of the auxiliar mesh $\mathcal{T}^{\prime}$.

\section{i) Auxiliar mesh $T^{\prime}$}

We start from a file which contains a set of points $(x, y, H(x, y))$. We have built this file from the level curves of a plane of the studied real domain.

The information that we have extracted from each level curve contains a set of points whose horizontal coordinates $(x, y)$ can be very close. So, we apply a filter to all the set of points. The idea is to construct a rectangular mesh and to choose just 
one point in each rectangle, the nearest point to the "center of mass" of all points contained in the rectangle.

With this new set of points we define a mesh of triangles, in such a way that each vertex of each triangle is a point of this set. The purpose of the previous filter is to avoid the formation of degenerated triangles in this mesh.

We will refer to this mesh as $\mathcal{T}^{\prime}$. We shall use this mesh to interpolate the bottom topography.

\section{ii) Triangular mesh $\mathcal{T}$}

Using the set of points and the topographic plain, we define a boundary of the domain. With this definition of the boundary, we can use a meshing program (in our case emc2 from INRIA) to obtain a mesh of triangles of the domain. Defining the number of points over the boundary we can control the size of the triangles.

We also use mesh adapting techniques (see [6]) in order to make the triangles smaller over the dam and in areas with a big gradient of topography.

We denote the final triangulation mesh by $\mathcal{T}$. This triangulation $\mathcal{T}$ is used in Subsection 6.3 to define the edge type finite volume mesh.

\section{iii) Interpolation of the topography}

To each node of the mesh $\mathcal{T}$ we must associate a value of the topography.

The process of interpolation has been done as follows: at first, for a given vertex of the triangulation $\mathcal{T}$, we look for the triangle of $\mathcal{T}^{\prime}$ in which it is contained. Subsequently, as we know the bottom topography on each vertex of $\mathcal{T}^{\prime}$, we interpolate using the barycentric coordinates of the vertex in a triangle of $\mathcal{T}^{\prime}$ : if a given vertex $v_{i}$ of $\mathcal{T}$ is contained in a triangle $T$ of $\mathcal{T}^{\prime},\left(\lambda_{1}, \lambda_{2}, \lambda_{3}\right)$ are its barycentric coordinates on $T$ and $p_{1}, p_{2}, p_{3}$ are the corresponding bottom topography at the nodes of $T$, we interpolate the bottom function at $v_{i}$ by

$$
\lambda_{1} p_{1}+\lambda_{2} p_{2}+\lambda_{3} p_{3}
$$




\section{Appendix B: Notation}

\begin{tabular}{||ll||}
\hline$|A|$ & absolute value of matrix $A$ \\
$A^{-1}$ & generalized inverse matrix of A (see Section 2) \\
$\mathcal{A}(W, \eta)$ & $F_{1}^{\prime} \eta_{1}+F_{2}^{\prime} \eta_{2}$ \\
$d_{i j}$ & upwinding matrix of the scheme \\
$F$ & distance between the nodes $N_{i}$ and $N_{j}$ \\
$F_{C}$ & physical flux function of the $1 \mathrm{D}$ problem \\
$\mathbb{F}$ & centered approximation of $F$ \\
$\mathbb{F}_{C}$ & centered approximation of $\mathbb{F}$ \\
$G$ & source term \\
$G_{C}$ & centered approximation of $G$ \\
$G_{D}$ & approximation of $G$ at a point in the boundary of a control volume \\
$\widetilde{G}_{(\xi, U)}$ & $G(x(\xi), U)$, where $x(\xi)$ is a line in the $2 \mathrm{D}$ domain (see Section 3$)$ \\
$\widetilde{G}_{i j}(\xi, U)$ & $G\left(P_{i j}+\xi \eta_{i j}, U\right)$ \\
$\widehat{G}_{i j}$ & approximation of $\widetilde{G}_{i j}$ at $\xi=0$ \\
$h$ & height of the water column \\
$H$ & bathimetry: $H=\bar{A}-z_{b}$, where $\bar{A}$ is a reference level \\
$I$ & identity matrix \\
$K_{i}$ & set of indices of the control volumes neighboring $V_{i}$ (see Section 3 ) \\
$N_{i}$ & center point of the control volume $V_{i}$ \\
$P_{i j}$ & point of $\Gamma_{i j}$ \\
$q_{1}, q_{2}$ & discharges on $x$ and $y$ directions, respectively \\
$t$ & time variable \\
$V_{i}$ & a control volume of a finite volume mesh \\
$\left|V_{i}\right|$ & area of $V_{i}$ \\
$V_{i j}$ & triangle interior to $V_{i}$ defined by $N_{i}$ and $\Gamma_{i j}$ \\
\hline \hline
\end{tabular}




\begin{tabular}{||ll||}
\hline \hline$\left|V_{i j}\right|$ & area of $V_{i j}$ \\
$W$ & vector of conservative unknowns \\
$W_{i}$ & approximation of the mean value of $W$ over the control volume $V_{i}$ \\
$W_{(1-\alpha) i+\alpha j}$ & $(1-\alpha) W_{i}+\alpha W_{j}$ \\
$X$ & matrix whose columns are the eigenvectors of $\mathcal{A}(W, \eta)$ \\
$x, y$ & horizontal space variables \\
$\mathbf{x}$ & (x, y) \\
$z_{b}$ & bottom function \\
$\Delta x, \Delta t$ & space and time steps \\
$\phi$ & numerical flux function for the homogeneous problem \\
$\phi_{G}$ & numerical flux function depending on $G$ \\
$\gamma_{l}$ & edges of $V_{i j} l=0,1,2($ Figure 2$)$ where $\gamma_{0} \equiv \Gamma_{i j}$ \\
$\Gamma_{i j}$ & common edge to the volumes $V_{i}$ and $V_{j}$ \\
$\left\|\Gamma_{i j}\right\|$ & length of $\Gamma_{i j}$ \\
$\eta_{i j}$ & unitary vector ortogonal to $\Gamma_{i j}$ pointing from $V_{i}$ to $V_{j}$ \\
$\lambda_{i j}$ & eigenvalues of $\mathcal{A}\left(\frac{W_{i}+W_{j}}{2}, \eta_{i j}\right)$ \\
$\Lambda$ & diagonal matrix of the eigenvalues of $\mathcal{A}(W, \eta)$ \\
$\nu$ & $\Delta x / 2$ \\
$\nu_{i j}$ & $d_{i j} / 2$ \\
$\rho$ & $\frac{1}{2}+\alpha(1-\alpha)$ with $\alpha \in[0,1]$ \\
\hline \hline
\end{tabular}

\section{Conclusions}

We have introduced in this paper a systematic technique to extend finite volume solvers for $2 \mathrm{D}$ hyperbolic systems to non-homogeneous systems. To do this we proceed similarly to the derivation of standard finite volume solvers using the line method: we project the system (now including the source terms) along the normal to the boundary segment between two neighboring cells, assuming that the source terms only vary on the normal direction.

We then apply 1D well-balanced approximated Riemann solvers to approximate the resulting problems.

By this procedure we obtain a large family of conservative solvers that we prove to be asymptotically well-balanced for essentially 1D steady solutions, on grids which are compatible with the solution. In addition, we give general conditions that we prove to be sufficient for a given scheme to exactly solve an actual steady solution at grid nodes. A relevant feature of these equilibrium properties is that we separately balance the centered and the de-centered part of our schemes.

We apply this general theory to 2D SWE, for which we introduce a systematic discretization of source terms, in particular those coming from variable bottom topography. We also introduce a large family of schemes that we prove to be asymptotically well-balanced for essentially 1D solutions, and to exactly solve water at 
rest.

Although by now we are not able to give a proof of stability of our method or error estimates, in practice our schemes present good accuracy and stability properties in tests involving strong shocks, rarefaction waves, sharp bottom gradients and large transient wet/dry transitions.

Finally we have applied the best of the schemes introduced to the ecological disaster produced by the dam break of the mining ponds in Aznalcóllar happened in Spain in 1998. The good properties of the introduced solver is confirmed by this hard test.

\section{Acknowledgments.}

We thank Prof. Francisco J. Segovia Espiau for interesting discussion on the application to the real dam break of Aznalcóllar and the topographical data.

This research has been partially supported by the Spanish Government Research projects MTM2006-08075 and MTM2006-01275.

\section{References}

[1] Vadym Aizinger, Clint Dawson, The local discontinuous galerkin method for three-dimensional shallow water flow. Comput. Methods Appl. Mech. engrg. 196, 734-746 (2007)

[2] M. Amara, D. Capatina-Papaghiuc, D. Trujillo, Approximation of New 2D and 1D hydrodynamical Models. Eccomas 2004 CD-RON Proceedings.

[3] E. Audusse, M. Bristeau, B. Perthame Second order kinetic scheme for SaintVenant equations with source terms. Eccomas 2004 CD-RON Proceedings.

[4] A. Bermúdez, A. Dervieux, J. A. Desideri, M. E. Vázquez Cendón, Upwind schemes for the two-dimensional shallow water equations with variable depth using unstructured meshes. Comput. Methods Appl. Mech. Eng. 155,49 (1998).

[5] A. Bermúdez - M. E. Vázquez Cendón, Upwind Methods for Hyperbolic Conservation Laws with Source Terms. Computers Fluids 23-8 1049-1071 (1994).

[6] M.J. Castro, Generación y adaptación anisótropa de mallados de elementos finitos. Aplicación a la resolución numérica de E.D.P. Universidad de Málaga (1996).

[7] M.J. Castro, A.M. Ferreiro, J.A. García, J.M. González, J. Macías, C. Parés, M.E. Vázquez, On the numerical treatment of wet/dry fronts in shallow flows: 
application to one-layer and two-layers systems. Match Comp. Model. 42 (34): 419-439 (2005).

[8] M.J. Castro, J.M. González-Vida, C. Parés, Numerical treatment of wet/dry fronts in shallow flows with a modified Roe scheme. Math. Mod. Meth. App. Sci. Vol. 16, No. 6, 897-931 (2006).

[9] M. J. Castro, J. A. García , J. M. González, C. Pares, A parallel $2 D$ finite volume scheme for solving systems of balance laws with nonconservative products: application to shallow flows.. Comput. Methods Appl. Mech. Eng. 19-22, 2788-2815 (2006).

[10] P. Brufau. Simulación bidimensional de flujos hidrodinámicos transitorios en gemotrías irregulares. Ph. D. Thesis Universidad de Zaragoza (2000).

[11] P. Brufau, P. García-Navarro, Unsteady free surface flow simulation over complex topography with a multidimensional upwind technique. J. Comput. Phys., 186, 503-526 (2003).

[12] W. Carlisle Thacker, Some exact solutions to the nonlinear shallow-water wave equations. J. Fluid Mech. 107, 499-508 (1981).

[13] M.J. Castro, T. Chacón Rebollo, E.D. Fernández-Nieto, C. Parés, On Balanced Finite Volume methods for non-homogenous hyperbolic systems with non-conservative terms.. SIAM J. Sci. Comput. 29 no. 3, 1093-1126 (2007).

[14] T. Chacón Rebollo, A. Domínguez Delgado, E.D. Fernández-Nieto, Asymptotically balanced schemes for non-homogeneous hyperbolic systems. Application to the Shallow Water Equations. C. R. Acad. Sci. Paris, Ser. I 338, 85-90 (2004).

[15] G.Dal Maso, P.G.LeFloch, F.Murat., Definition and weak stability of nonconservative products. J. Math. Pures Appl. 74: 483-548, (1995).

[16] T. Gallouët, J.-M. Hérard, N. Seguin, Some approximate Godunov schemes to compute shallow-water equations with topography. Computers \& Fluids 32 479-513 (2003).

[17] E. Godlewski - P. A. Raviart, Numerical Approximation of Hyperbolic Systems of Conservation Laws. Springer - Verlag (1996).

[18] J.M. Greenberg, A. Y. Leroux. A Well-Balanced scheme for the numerical processing of source terms in hyperbolic equations.. SIAM J. Numer. Anal. Vol. 33 No. 1, pp. 1-16 (1996).

[19] J.M. Hervouet, Hydrodynamique des Ecoulements à surface libre. Presses de l'Ecole Nationale des Ponts et Chaussées (2003). 
[20] R. Holdahl, H. Holden, Knut-A. Lie, Unconditionally stable splitting methods for the shallow water equations BIT 39, n. 3, 451-472 (1999).

[21] A. Kurganov, D. Levy, Central-upwind schemes for the saint-venant system. M2AN, Vol. 36, N. 3, 397-425 (2002).

[22] R. J. Le Veque, Balancing Source Terms and Flux Gradients in HighResolution Godunov Methods: The Quasi-Steady Wave-Propagation Algorithm. J. Comp. Phys. 146, 346-365, (1998).

[23] C. Parés, M.J. Castro., On the well-balanced property of Roe method for nonconservative hyperbolic systems. Applications to shallow water systems. ESAIM: M2AN, Vol 38(5): 821-852, (2004).

[24] B. Perthame, C. Simeoni, A kinetic scheme for the Saint-Venant system with a source term. Calcolo 38 n. 4, 201-231 (2001).

[25] M. Quecedo, M. Pastro, M.I. Herreros, J.a. Fernández Merodo, Qinfen Zhang, Comparison of two mathematical models for solving the dam break problem using the FEM method. Comput. Methods Appl. Mech. Engrg. 194, 3984-4005 (2005)

[26] P. J. Roache, Perspective: a method for uniform reporting of grid refinement studies. ASME J Fluids Eng. 116, 405-413 (1994).

[27] P. L. Roe, Upwind differencing schemes for hyperbolic conservation laws with source terms. Nonlinear Hyperbolic Problems, C. Carraso, P.A. Raviart, D. Serre, eds. Springer-Verlag, Lecture Notes in Mathematics 1270, 41-51. (1986).

[28] P. L. Roe. Approximate Riemann solvers, parameter vectors, and difference schemes. J. Comput. Phys., 43 , 357-371 (1981).

[29] E. F. Toro. Riemann Solvers and Numerical Methods for Fluid Dynamics. Springer (1997).

[30] E.F. Toro. Shock-Capturing Methods for Free-Surface Shallow Flows. Whiley (2001).

[31] J. G. Zhou, P.K. Stansby, 2D Shallow Water Flow Model for the Hydraulic Jump. int. J. Numer. Meth. Fluids 29, 375-387 (1999).

[32] J. G. Zhou, D.M. Causon, C.G. Mingham, and D. M. Ingram, The Surface Gradient Method for the Treatment of Source Terms in the Sallow-Water Equations. J. Comput. Phys. 168, 1-25 (2001). 